\title{
On the Role of Thermal Boundary Conditions in Typical Problems of Buoyancy Convection: A Combined Experimental-Numerical Analysis
}

\author{
Hermes Ferialdi, Marcello Lappa and Christopher Haughey ${ }^{1}$ \\ ${ }^{1}$ Department of Mechanical and Aerospace Engineering, University of Strathclyde, James Weir \\ Building, 75 Montrose Street, Glasgow, G1 1XJ, UK - email: marcello.lappa@strath.ac.uk
}

\begin{abstract}
Buoyancy flows of thermal origin and related heat transfer problems are central in a variety of disciplines and technological applications. In the present study the classical case of a cavity heated from one side and cooled from the other (internal size $4 \mathrm{~cm}$, filled with water) is tackled both experimentally and numerically for different circumstances (horizontal and inclined temperature gradients). The main objective is to fill a gap, namely, the surprising lack of knowledge relating to the role played by the effective heat loss taking place through the walls delimiting the fluid domain from above and from below and along the spanwise direction in influencing the instabilities of these flows and their progression towards chaos. We explore the response of such systems with respect to several parameters, including the inclination of the cavity with respect to gravity, the average temperature of the fluid, the applied temperature difference, the dependence of fluid properties on temperature and the intensity of heat transfer to the ambient. Experiments are supported by dedicated numerical simulations based on the Navier Stokes and energy equations in their time-dependent and non-linear formulation (solved by means of the SIMPLE method with a collocated-grid approach). It is shown that a kaleidoscope of states is possible depending on the considered conditions. The results reveal the counter-intuitive triadic relationship among heat loss through non-thermally active walls, the hierarchy of bifurcations displayed by the system and the prevailing two-dimensional or three-dimensional nature of the flow.
\end{abstract}

Key words: Buoyancy flow, water, heat exchange, instability and bifurcation in fluid dynamics.

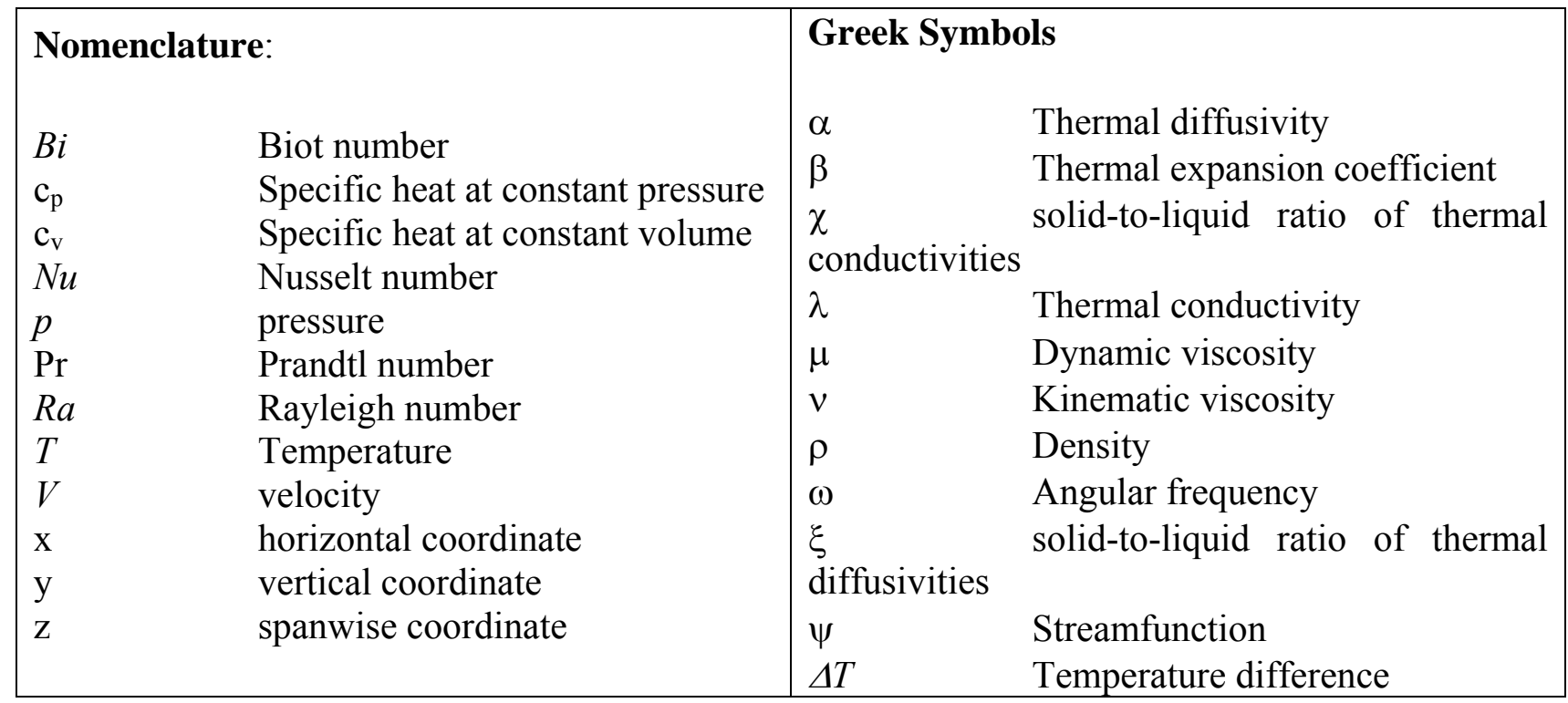




\begin{tabular}{|ll|ll|}
\hline Subscripts & & \\
0 & reference conditions & Superscripts \\
Amb & ambient & Air & air \\
Back & wall delimiting the system along the spanwise direction & Liquid & liquid \\
Bottom & bottom & Solid & solid \\
Cold & cold & \\
eff & effective & \\
Front & wall delimiting the system along the spanwise direction & \\
Hot & hot & \\
REF & reference & \\
Top & top & \\
Wall & wall & \\
& & \\
\hline
\end{tabular}

\section{Introduction}

Flows induced by buoyancy convection in non-isothermal fluids are widespread in nature and in technology (Melnikov and Shevtsova [1]; Kaddeche et al. [2]; Delgado Buscalioni et al. [3]; Sun and Jaluria [4]; Martineau et al. [5]; Bontoux et al. [6]; Bennacer et al. [7]; Hadidi and Bennacer [8]; Sohail and Saghir [9]; Lappa [10], Soltani and Rahal [11]; Azoui et al. [12]). This is the reason why this research area has attracted so much attention since the seminal model of circulation introduced by Hadley [13] approximately three hundred years ago.

This theme is so rich that it has been approached under very different perspectives (and with completely different motivations) by several research groups operating in disparate fields such as: fundamental physics (see, e.g., Cross and Hohenberg [14]), meteorology (Emanuel [15]; Thorpe [16]), oceanography, geophysics (Richter, [17]; Woods and Lintz [18]), astrophysics (Heng and Showman [19]), the science of materials (Lappa [20-22], Gelfgat et al. [23]; Sohail and Saghir [9]), environmental control systems and heat exchangers ([4,5,7,8], biology (Benoit et al. [24]), etc.

The similarity accompanying all these subjects with respect to the underlying physical mechanisms and the overarching mathematics has led over the years to establishing a common framework that is now generally referred to as the field of 'thermal convection' (Lappa [25]). More precisely, such a commonality has led to the definition of general objectives and a general modus operandi. As an example, there is a long tradition of studies dealing with the investigation of buoyancy convection in closed cavities for different relative direction of the imposed temperature gradient $(\Delta T)$ and gravity $(\mathrm{g})$ as it is expected that this factor can play a vital role in determining the features of the emerging flow. When the angle formed by $\Delta T$ and $\mathrm{g}$ is zero or 180 degrees, the problem reduces to a canonical configurations widely investigated in the literature, namely standard Rayleigh-Bénard (RB) convection (for which $\mathrm{g}$ and $\Delta \mathrm{T}$ are concurrent) or to thermally diffusive and quiescent conditions (g and $\Delta T$ antiparallel). Rotating gravity by 90 degrees leads to another archetype, generally known as the Hadley flow [13]. While the typical outcomes of these three fundamental situations are parallel rolls (parallel $\mathrm{g}$ and $\Delta \mathrm{T}$ ), gravity waves (opposite $\mathrm{g}$ and $\Delta T$ ) and unicellular flow ( $\mathrm{g}$ and $\Delta \mathrm{T}$ perpendicular), respectively, the complexity of the problem dramatically increases when intermediate inclinations are considered. 
The common way of thinking (or of approaching these problems) outlined above, however, is not limited to fixing the inclination of gravity with respect to the imposed temperature difference and investigating the response of the system for growing values of $\Delta T$ (i.e., the typical hierarchy of bifurcations). The various analyses reported in the literature can also be categorized in terms of the specific boundary conditions considered by investigators in either experimental or numerical studies on the subject. While the temperature is generally assumed to be constant and fixed along the thermally-active walls (namely on the walls where the temperature is actively controlled), other conditions apply to the remaining solid boundaries (the non-thermally active ones, i.e. those where temperature is not imposed). These are generally assumed to be perfectly adiabatic or conducting, the former situation corresponding to zero heat flux, the latter resulting in a temperature that varies linearly along the wall (temperature increasing linearly from the minimum temperature in the system towards its maximum value). Though these conditions might be regarded as purely mathematical idealizations, they have actually been used to classify a vast amount of results, including experimental ones.

This modus operandi should not necessarily be seen as a drawback. By putting a constraint on the degrees of freedom of the considered system, the use of idealized models and simplified boundary conditions can lead, with no doubt, to more efficient exploration of the space of parameters, especially in the context of theoretical and numerical studies [10,25]. Indeed, following this approach many authors could examine the stability of the Hadley flow in infinite layers and produce several valuable results. Given the importance of these results, although the current work is focused on finite-sized cavities, in the following we conduct a short review of them (this being helpful for purposes of reference and for introducing in a relatively simple way the main known fundamental mechanisms of instability in these systems).

Seminal linear stability analyses (LSA) focusing on the Hadley convection are due to Hart [26,27], who examined its sensitivity to disturbances oriented along the streamwise or spanwise direction (referred to as transverse and longitudinal perturbations, respectively). The former (a twodimensional disturbance) is a consequence of inertial forces (the shear-driven nature of the flow), while a dynamical balance between the inertial and gravitational forces is at the root of the latter category of (helicoidal, i.e. three-dimensional) disturbances (Gill [28]). Leaving aside for a while the spatial structure, these convective modes also display notable differences in terms of temporal behavior, with transverse and longitudinal disturbances leading to steady and oscillatory multicellular flows, respectively [26-28].

Notably, all these studies created a sort of 'dichotomy' between situations supporting the amplification of one or the other class of disturbances. The ranges of existence of these classes were found to depend on the considered fluid and the thermal conditions established at the top and bottom solid walls. However, it is worth noticing that, since both categories were always observed in a range of values of the Prandtl number (Pr) smaller that the unit order of magnitude $(\operatorname{Pr} \leq \mathrm{O}(1))$, these theoretical results were essentially used (from a historical point of view) to interpret the behaviors of liquid metals (Kuo and Korpela [29]). 
It should be pointed out, however, that a third category of disturbances also exists for the specific case of conducting walls and fluids with significantly larger values of the Prandtl number. Related findings, (obtained through linear stability analysis including the range of high Prandtl numbers $\operatorname{Pr} \geq \mathrm{O}(1)$ ) can be found in the study by Gershuni et al. [30]. Due to this line of inquiry, eventually, it has been recognized that the presence of zones of potentially unstable stratification near the upper and lower horizontal conducting boundaries (induced by the basic flow) can lead to convection of the Rayleigh-Bénard type therein. This flow typically emerges in the form of steady rolls with their axes aligned with the shear flow (i.e. longitudinal rolls), or (at slightly larger values of $\mathrm{Ra}$ ) as oscillatory rolls with their axes perpendicular to the shear flow (two-dimensional oscillatory Rayleigh mode). According to Gershuni et al. [30], these modes exist for $\operatorname{Pr}>0.44$ and remain the most dangerous up to extremely large Pr.

Interestingly, this specific type of disturbances become even more relevant if configurations with a temperature gradient inclined with respect to the horizontal direction are considered (DelgadoBuscalioni [31]). In such cases, indeed, due to the inclination, a destabilizing component of the temperature gradient along the vertical direction can be present even if the non-thermally active walls are perfectly adiabatic. Remarkably, this led some authors, e.g., Hart [32] and DelgadoBuscalioni [31] to the identification of a zoo of possible alternate modes of convection, which are not possible with no inclination.

We conclude this focused review of the literature (all the arguments presented here will be placed in a more relevant context in Sect. 4 where we will discuss configurations of finite extent) by observing that a forth general category of fluid-dynamic instabilities is made possible in finite-sized systems just by the existence of lateral walls. The thermal boundary layers developing along these walls can indeed provide the background for the amplification of other types of perturbations for $\mathrm{Pr}$ $\geq \mathrm{O}(1)[31]$.

As the reader might have realized at this stage, there is no doubt that all these results have helped investigators to discern important mechanisms of instability, often used to interpret practical problems. Nevertheless, we wish to highlight that a residual gap still exists. It concerns cases where a departure from the idealized situations of adiabatic and conducting boundaries occurs.

Indeed, the curse represented by the effective heat exchange taking place through non-thermally active walls is always present in practical problems and it might be a serious shortcoming for further advances (especially if they have to be based on meaningful comparison of theoretical/numerical and experimental results).

Along these lines, the research scheme implemented in the present work envisions a parametric hybrid experimental/numerical analysis aimed to evaluate the impact of the effective heat exchange taking place through non-thermally active walls on the hierarchy of bifurcations characterizing the buoyancy flow in the fundamental situations described before (namely, horizontal or inclined differentially heated cavities). We also quantify the departure from the conditions established when idealized thermal boundary conditions are implemented. 
In particular, while the angle between the imposed temperature difference and gravity is varied using a dedicated facility, we intentionally change the magnitude of the heat losses through the walls by modifying the average temperature of the fluid (while keeping constant the ambient temperature). The Rayleigh number based on the temperature difference between the hot and cold walls is also kept constant in order to assess precisely the location of the system in such conditions along the path ideally bringing it from the initial steady state towards chaos. Dedicated twodimensional and three-dimensional numerical simulations are conducted in the framework of a finite-difference approach, expressly taking into account the dependence of the fluid properties on temperature and the heat flowing through the walls towards the external ambient.

Such a parametric investigation is carried out with the explicit intent to help investigators to discern the interrelations among the various parameters under one's control (which are not independent) and their impact on the emerging patterns and states (steady, time-periodic or turbulent).

\section{Experimental Apparatus and Procedure}

In order to study different mechanisms of thermal-convection highlighted in the introduction, a dedicated facility has been developed, i.e. the TCFS (Thermal Convection Fluid Science) Test Rig. This facility supports the use of various Perspex 'boxes' with different sizes and aspect ratios, hereafter simply referred to as "Fluid Containers" (FCs), upon which a variety of conditions can be tested in terms of thermal stimuli and buoyancy effects. To study different forms of thermal convection, the apparatus has been designed to incline the FCs above the horizontal direction up to $180^{\circ}$ i.e. upside-down.

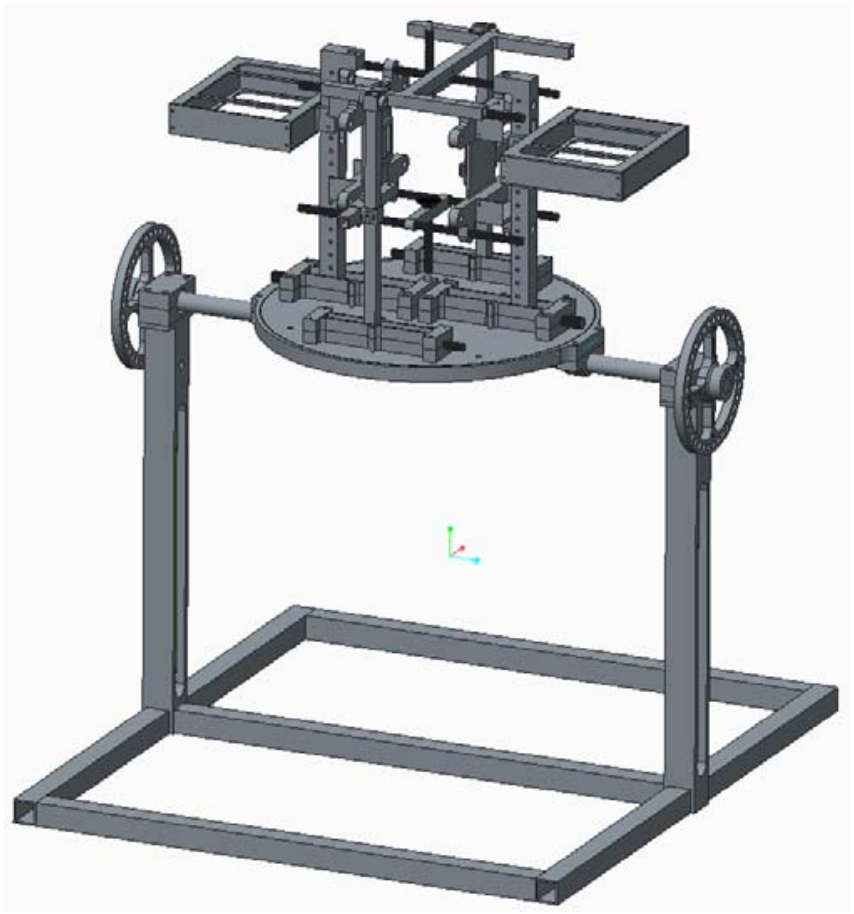

Figure 1: CAD model of the original design of the TCFS Test Rig. 
Additional details can be gathered from Fig.1, where it is evident that the TCFS Test Rig can be seen as the combination of two large sub-assemblies, namely the Rotating Base Assembly (having horizontal dimensions $0.84 \times 0.56 \mathrm{~m}$ and height $0.49 \mathrm{~m}$, used to support the facility and allow variation of the angle of inclination) and the Thermal Systems Assembly (providing the necessary functionality to establish temperature gradients of desired strength across each of the FCs). The rig also includes the Fan Support Assemblies (FSA) with adjustable height (maximum height $0.82 \mathrm{~m}$ ), required for efficient heat removal from the Cooling System Radiators. The FSA are isolated with respect to the main body of the facility in order to avoid the propagation of disturbing vibrations from the fan to the fluid containers.

Though this system displays an intrinsic degree of flexibility allowed by the possibility to study thermal-convection for different relative directions of the imposed temperature difference and the direction of gravity, it can be used in two "fundamental" or "basic" operative modes: a vertical configuration and a horizontal configuration. The overall apparatus can be reconfigured in either one of these configurations according to the problem of interest, be it a situation in which the dominant temperature gradient is vertical or horizontal.

The FC is constrained between two opposing Cooling System Containers (CSCs), each of which includes a Peltier Module and liquid-based CPU cooler. Moreover, each CSC is installed on two Support Frames with position adjustable depending on the size of the FC being supported. A good thermal contact is made possible by the application of silicone gel, which prevents a layer of insulating air from being formed between these components. Although the FC is contained between the two CSCs, when the TCFS is in the horizontal configuration, the weight of the FC is supported by two pneumatic suction pads which are suspended on rods between the CSCs - a $12 \mathrm{~V}$ vacuum pump is used to provide a vacuum of approximately 16 " $\mathrm{Hg}$. The suction pads, which can be adjusted according to the dimensions of the FC, can then be applied to any two of the four remaining faces of the FC (two of the faces are occupied by the CSCs). This gives the operator the ability to position a Microscope or a simple camera against a number of different faces of the FC, therefore making it possible for the fluid to be filmed from several perspectives. However, it is not possible to mount the Microscope (or camera) against the bottom face of the FC. Therefore, once the FC is mounted on the Test Rig in the horizontal configuration, two faces of the FC are occupied by the CSCs, another two by the suction pads, and the final two faces are occupied by the Microscope and the Light Source. In the Horizontal Configuration, both CSCs are attached by screws to their respective Support Frames. If the operator wishes to record the fluid from a different perspective, the TCFS Test Rig can be rotated about the FC to allow the Microscope to be sited against another face, again ensuring that the observer has the ability to interrogate the fluid behavior from a number of angles. 


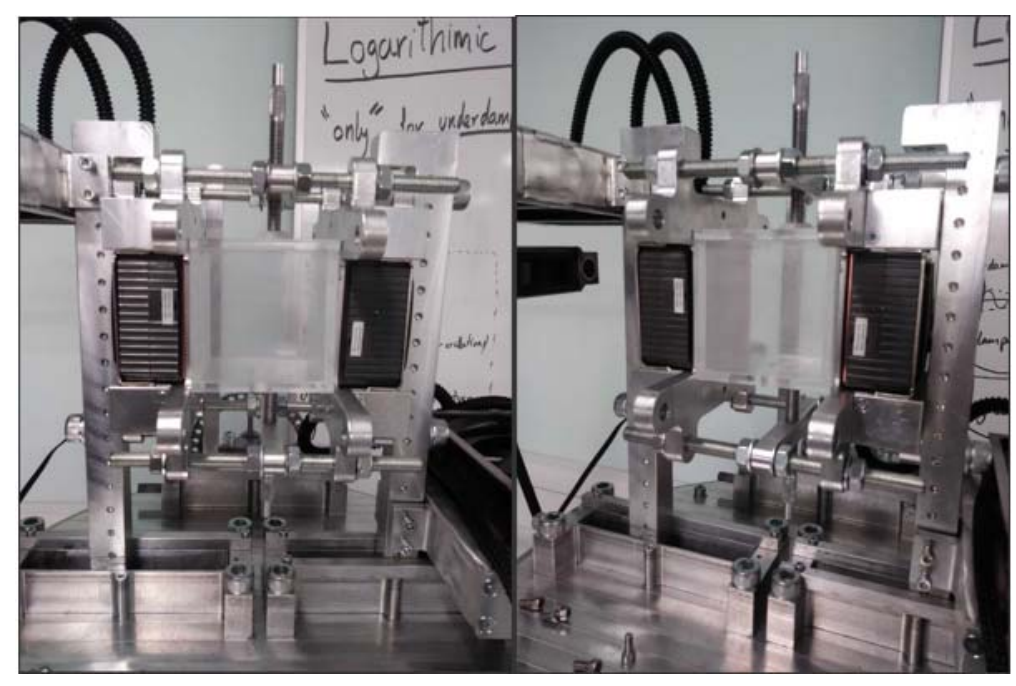

Figure 2: TCFS Test Rig with the $60 \times 60$ × $60 \mathrm{~mm}$ Perspex FC. Note that the Peltier Brackets, Peltier Devices and associated wiring have been removed for clarity:

The TCFS Test Rig can be operated together with three cubic and cuboidal FCs with various inner dimensions, namely $40 \times 40$ x $40 \mathrm{~mm}$ (used for the present experiments), $60 \times 60 \times 60 \mathrm{~mm}$ or $40 \mathrm{x}$ $40 \times 90 \mathrm{~mm}$. The temperature gradient required to put the fluid in motion is generated by two opposing Peltier Modules (40 mm x $40 \mathrm{~mm}$ x $4 \mathrm{~mm}, 12 \mathrm{~V}, 6 \mathrm{~A}$ ) connected to a Omega CN32PT440-DC PID Temperature Controller (accurate to $+/-0.2^{\circ} \mathrm{C}$ ) relying on a $5 \mathrm{k} \Omega$ thermistor (accurate to $+/-0.1^{\circ} \mathrm{C}$ ) put directly in contact with the face of the $\mathrm{FC}$ to determine the temperature. Therefore the overall temperature accuracy on the outer surface of the $\mathrm{FC}$ is $+/-0.3^{\circ} \mathrm{C}$. Despite the Temperature Controller is equipped with an inbuilt self-tuning function, it was found that, for temperatures smaller than $70^{\circ} \mathrm{C}$, the PID parameters automatically set by the controller were not sufficiently accurate. For this reason, some readjustment of the PID parameters had typically to be implemented manually before starting any experiment. With such strategy, a temperature deviation less than $+/-0.1^{\circ} \mathrm{C}$, and often as low as $+/-0.02^{\circ} \mathrm{C}$, could be obtained.

Continuing with the description of the facility and its sub-systems, it is worth pointing out that, in practice, the DC pulse signals generated by the Temperature Controller are transferred to a Solid State Relay (SSR) connected with the Peltier Module to form a simple series circuit (Fig. 3). The SSR essentially transforms the sinusoidal output of the Temperature Controller into a square wave "on-off" signal. In this way the voltage supplied to the Peltier Devices can be modulated in order to maintain a desired temperature.

As outlined before, heat is extracted from each Peltier Module using a Cooler Master Seidon 120V Version 2.0. This system is based on a special fluid being continuously pumped between a copper plate and a radiator. The nominal power rating of the Cooling System indicates that each Cooling System can dissipate up to around 300W. Moreover, as the shape of the CSCs is relatively extended in space, heat can be removed from the Peltier Devices in an efficient way. Along these lines, dedicated test activities have led to the conclusion that the TCFS Test Rig is able to maintain a 
constant temperature as high as $100^{\circ} \mathrm{C}$ at the outer FC surface. Vice versa, constant temperatures as low as $10^{\circ} \mathrm{C}$ could also be obtained.

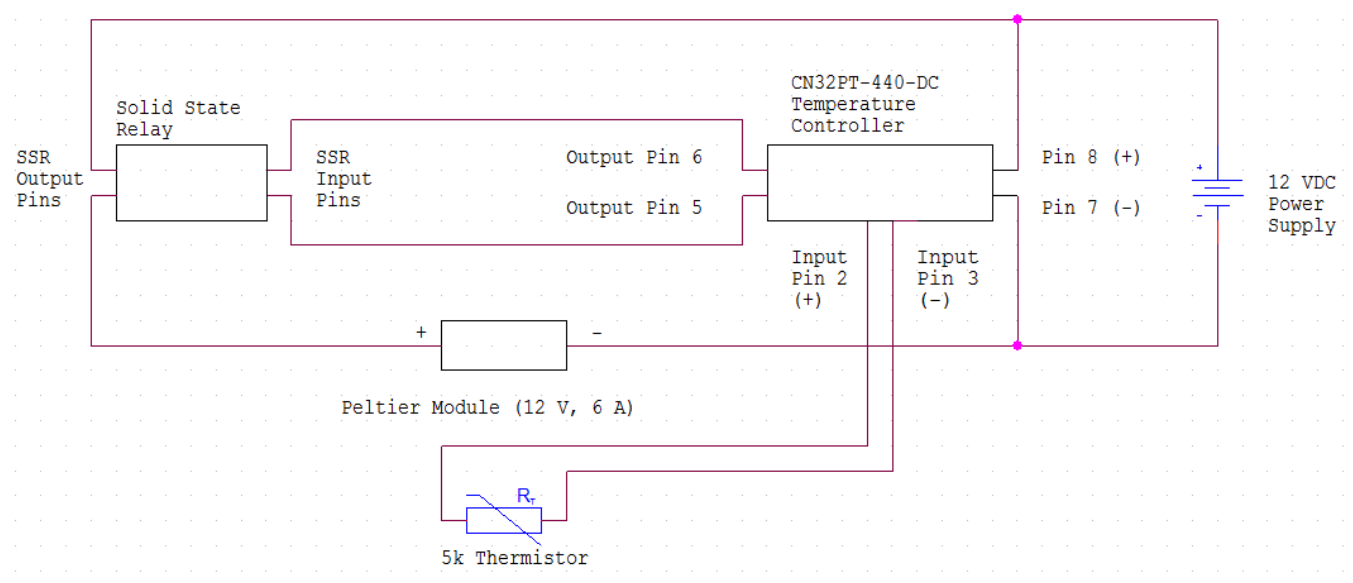

Figure 3: Circuit Diagram of the Temperature Control Circuit of the TCFS Test Rig. Overall, there are two such circuits i.e. one for each Peltier Device.

Technical sheet: TCFS (Thermal Convection Fluid Science) Test Rig

\begin{tabular}{|c|c|}
\hline Component & Properties / Performances \\
\hline 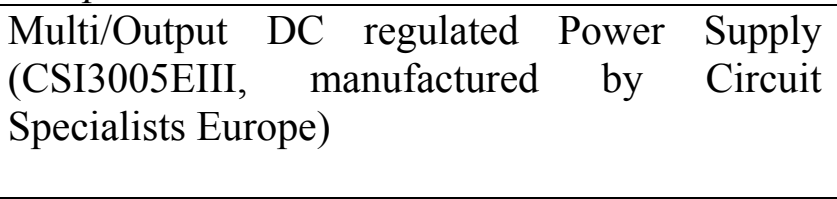 & $\begin{array}{l}\text { Output voltage: } 0-30 \mathrm{~V} \text { (adj. continuously) } \\
\text { Output current: } 0-5 \mathrm{~A} \text { (adj. continuously) } \\
\text { Volt-indication: } \quad \text { LED } \pm 1 \% \pm 2 \text { digits } \\
\text { Amp-indication: } \quad \text { LED } \pm 2 \%\end{array}$ \\
\hline $\begin{array}{l}\text { Temperature controller (CN32PT-440-DC PID, } \\
\text { manufactured by OMEGA) }\end{array}$ & Accuracy $+/-0.2^{\circ} \mathrm{C}$ \\
\hline Thermistor & $5 \mathrm{k} \Omega$, Accuracy $+/-0.1^{\circ} \mathrm{C}$ \\
\hline Peltier Modules & $\begin{array}{l}4 \times 4 \mathrm{~cm}, 72 \mathrm{~W} \\
\text { Temperature Range: }-10 \text { to } 100^{\circ} \mathrm{C} \text { at the outer FC } \\
\text { surface }\end{array}$ \\
\hline $\begin{array}{l}\text { Cooling System (Cooler Master Seidon 120V, } \\
\text { Version 2.0) }\end{array}$ & Able to dissipate power up to $300 \mathrm{~W}$ \\
\hline Rotating Base Assembly & Horizontal size: $0.84 \times 0.56 \mathrm{~m}$ and height $0.49 \mathrm{~m}$ \\
\hline Fan Support Assemblies (FSA) & Maximum height $0.82 \mathrm{~m}$ \\
\hline Fluid containers $(\mathrm{FC})$ & $\begin{array}{l}\text { Inner dimensions: } 40 \times 40 \times 40 \mathrm{~mm} \text { (used for the } \\
\text { present experiments), } 60 \times 60 \times 60 \mathrm{~mm} \text { or } 40 \times 1 \\
40 \times 90 \mathrm{~mm}\end{array}$ \\
\hline Laser & $\begin{array}{l}\text { wavelength } 532 \mathrm{~nm} \pm 10 \text { and power max output }< \\
50 \mathrm{~mW}\end{array}$ \\
\hline Camera & Resolution: 3840x2160 pixels \\
\hline
\end{tabular}

The motion of tracers dispersed in the considered liquid is recorded by a Digital Microscope (up to 50x zoom) or a standard camera with resolution 3840x2160 pixels and stored on a nearby PC. Tracers revealing fluid motion are illuminated by a Light Source - a laser with wavelength 532 
$\mathrm{nm} \pm 10$ and power max output $<50 \mathrm{~mW}$. Fully adjustable support mechanisms are used to support both the Microscope and Light Source. To allow the FC to be inclined above the horizontal direction, the apparatus rests upon a central rotating shaft - this shaft allows the angle of inclination to be altered in increments of $10^{\circ}$, thereby allowing the fluid behavior to be studied across a range of inclinations.

\section{Mathematical Model}

Obtaining a robust understanding requires a range of model types, varying from fundamental to much more elaborated ones, in which various effects can be enabled or disregarded and the results carefully scrutinized. This modus operandi is generally called a modelling hierarchy and its implementation is typically used to get further progress in the analysis of systems governed by nonlinear dynamics, especially thermal convection. The modelling hierarchy for the cases of interest in the present work, in terms of governing equations, boundary conditions, approximations and nondimensional parameters, is described in this section. As outlined in the introduction, the key motivating idea is to cause a change in the existing paradigm and leverage the current modelling capabilities in addressing the impact of various parameters and their interaction in practical situations.

\section{$\underline{3.1 \text { Geometry }}$}
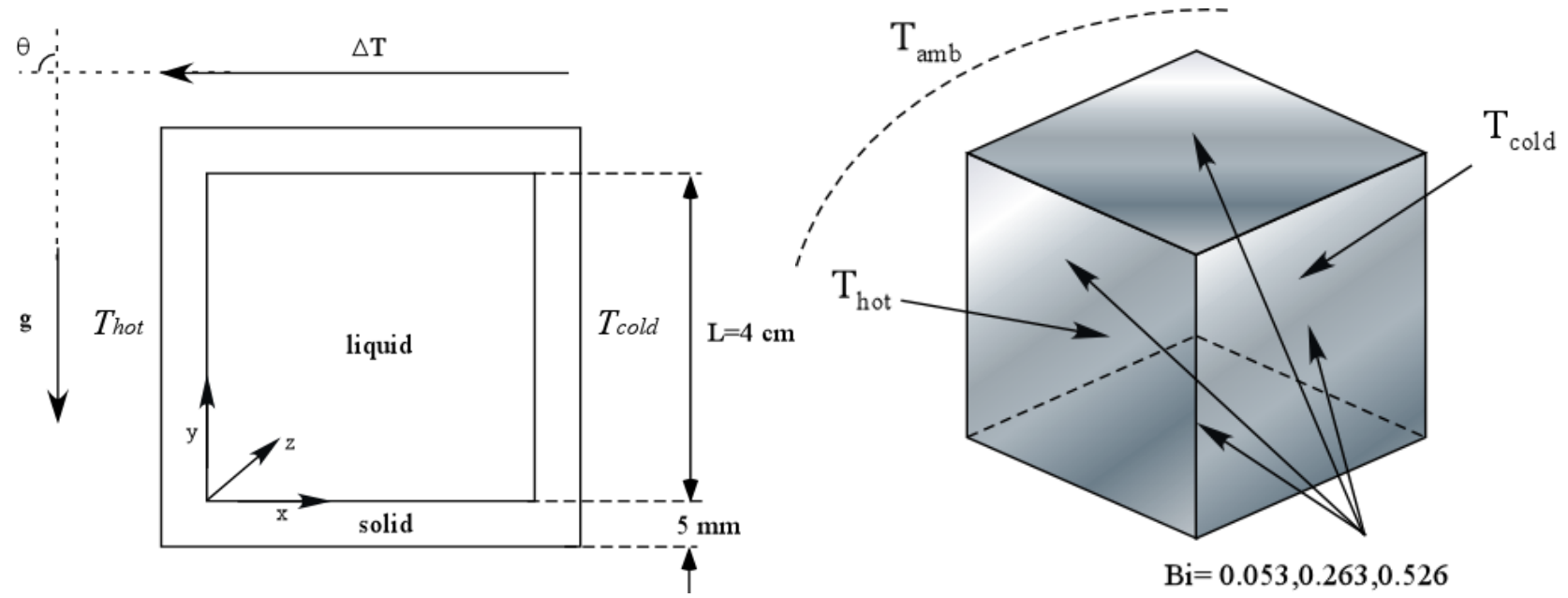

Figure 4: Geometry of the cavity with the perspex walls and related boundary conditions. The sketch on the left also shows the reference system (a classical Cartesian coordinate system with mutually perpendicular axes).

As explained in Sect. 1, the approach to the study of thermal convection adopted so far by the community of theoretical physicists and engineers has essentially been based on the paradigm of a series of different purely conceptual boundary conditions, i.e. adiabatic and conducting boundaries assumed to model the walls where no active control of temperature is implemented. 
The former would imply the use of materials with extremely low thermal conductivity (equal to zero theoretically speaking, corresponding to plastics or other insulating materials in practice), whereas the latter might be attained in the opposite case for which the thermal conductivity of the material is relatively high (practically realizable using walls made of a metal).

As materials with zero or infinite thermal conductivity, however, do not exist, these should be regarded as purely mathematical abstractions. This is the reason for which in the present study these idealized conditions are replaced by a more realistic framework in which we expressly account for the presence of the material (perspex) used to manufacture the walls of the FCs (solving the heat diffusion equation through the solid material) and the heat exchange with the ambient air. The model is sketched in Fig. 4.

Our explicit choice to use a container with all walls made of the same material resides in the intention to examine a 'minimal model' in which the fluid is encapsulated into a shell displaying homogeneous and isotropic properties along all directions, with heat transfer (controlled or uncontrolled) taking place at its external surface. Despite its apparent simplicity, with this configuration one is forced to account for what happens through the thickness of the solid walls (making the transport of heat in the homogeneous material a fundamental ingredient of the analysis, as further illustrated in the next section).

\section{$\underline{3.2 \text { Governing equations }}$}

The multi-domain approach used to deal with the present problems, where fluid and solid regions are properly coupled in terms of heat exchange, is described in the following.

\subsubsection{Fluid region}

In the liquid region, the classical Navier-Stokes equations for an incompressible fluid can be used. These consist of the balance equations for mass and momentum, properly enriched with the energy equation (required for problem closure). In dimensional form, this set can be cast in compact form as reported in the following:

$$
\begin{aligned}
& \underline{\nabla} \cdot \underline{V}=0 \\
& \rho_{R E F} \frac{\partial \underline{V}}{\partial \mathrm{t}}+\rho_{R E F} \underline{\nabla} \cdot[\underline{V} \underline{V}]+\underline{\nabla} p=\underline{\nabla} \cdot\left[2 \mu(\underline{\nabla} \underline{V})^{s}\right]+\rho \underline{g} \\
& \rho_{R E F} \frac{\partial\left(c_{v} T\right)}{\partial t}+\rho_{R E F} \underline{\nabla} \cdot\left[c_{v} \underline{V T}\right]=\underline{\nabla} \cdot\left[\lambda^{\text {liquid }} \underline{\nabla T}\right]
\end{aligned}
$$

where $\underline{V}$ is the fluid velocity, $p$ is its pressure, $T$ is the temperature, $\rho$ is the liquid density, $\mu$ is the dynamic viscosity, $\lambda$ is the thermal diffusivity, $c_{v}$ is the specific heat at constant volume, the 
subscript REF denotes a reference condition (corresponding to the temperature $T_{\mathrm{REF}}$ ) and $(\underline{\nabla} \underline{V})^{s}=\left(\underline{\nabla} \underline{V}+\underline{\nabla} \underline{V}^{T}\right) / 2$.

This formulation is a natural consequence of the initial assumption of constant density, which is generally regarded as a reliable approximation for a liquid, provided the dependence of density on temperature is not neglected in the buoyancy term (the source term at the right-hand side of the momentum equation). Density appearing there can formally be expressed via a Taylor series expansion accounting for its dependence on temperature, i.e.

$\rho=\rho(T)=\rho\left(T_{R E F}\right)+\left.\sum_{k=1}^{\infty} \frac{1}{k !} \frac{d^{k} \rho}{d T^{k}}\right|_{T_{R E F}}\left(T-T_{R E F}\right)^{k}$

In the present work we assume water as the work fluid. The reader may consider Table I for relevant information about the dependence of all the typical fluid properties on temperature for the case of water.

Table I: Physical properties of water as a function of temperature.

\begin{tabular}{|c|c|c|c|c|c|c|c|}
\hline $\begin{array}{c}T \\
{\left[{ }^{\circ} \mathrm{C}\right]}\end{array}$ & $\begin{array}{c}\lambda \\
{[\mathrm{W} /(\mathrm{mK})]}\end{array}$ & $\begin{array}{c}\rho \\
{\left[\mathrm{kg} / \mathrm{m}^{3}\right]}\end{array}$ & $\begin{array}{c}c_{p} \\
{[\mathrm{~J} /(\mathrm{KgK})]}\end{array}$ & $\begin{array}{c}\mu \\
{\left[K g /\left(m s^{2}\right)\right]}\end{array}$ & $\begin{array}{c}v \\
{\left[\mathrm{~m}^{2} / \mathrm{s}^{2}\right]}\end{array}$ & $\begin{array}{c}\alpha \\
{\left[\mathrm{m}^{2} / \mathrm{s}^{2}\right]}\end{array}$ & $\begin{array}{l}\operatorname{Pr} \\
{[/]}\end{array}$ \\
\hline 10 & 0.57864 & 999.77 & 4192 & 0.001308 & $1.308310^{-6}$ & $1.380710^{-7}$ & 9.4759 \\
\hline 20 & 0.59803 & 998.29 & 4182 & 0.001003 & $1.004710^{-6}$ & $1.432510^{-7}$ & 7.0139 \\
\hline 30 & 0.6145 & 995.71 & 4178 & 0.000798 & $8.014410^{-7}$ & $1.477110^{-7}$ & 5.4256 \\
\hline 40 & 0.62856 & 992.25 & 4179 & 0.000653 & $6.581010^{-7}$ & $1.515810^{-7}$ & 4.3415 \\
\hline 50 & 0.6406 & 988.02 & 4181 & 0.000547 & $5.536310^{-7}$ & $1.550710^{-7}$ & 3.5701 \\
\hline 60 & 0.65091 & 983.13 & 4185 & 0.000467 & $4.750010^{-7}$ & $1.582010^{-7}$ & 3.0026 \\
\hline 70 & 0.65969 & 977.63 & 4190 & 0.000404 & $4.132410^{-7}$ & $1.610010^{-7}$ & 2.5660 \\
\hline 80 & 0.66702 & 971.60 & 4196 & 0.000355 & $3.653810^{-7}$ & $1.636110^{-7}$ & 2.2332 \\
\hline 90 & 0.67288 & 965.06 & 4205 & 0.000315 & $3.264010^{-7}$ & $1.658110^{-7}$ & 1.9685 \\
\hline 100 & 0.67703 & 958.05 & 4216 & 0.000282 & $2.943510^{-7}$ & $1.676210^{-7}$ & 1.7561 \\
\hline
\end{tabular}

If eq. (4) is cut after the first-order derivative, i.e. $\left.\frac{d^{k} \rho}{d T^{k}}\right|_{T_{R E F}}$ is set to zero for all $\mathrm{k} \geq 2$ and the other thermodynamic properties appearing in the balance equations are assumed to be constant (namely, $\mu, \lambda$ and $c_{v}$ ), the considered formulation reduces to the so-called Boussinesq framework. Taking into account that for liquids $c_{v} \cong c_{p}$ (where $c_{p}$ is specific heat at constant pressure), the equations can be simplified in this case as:

$\underline{\nabla} \cdot \underline{V}=0$ 


$$
\begin{aligned}
& \frac{\partial \underline{V}}{\partial \mathrm{t}}+\underline{\nabla} \cdot[\underline{V} \underline{V}]+\frac{1}{\rho_{0}} \underline{\nabla} p=v_{0} \nabla^{2} \underline{V}+\left[1-\beta_{T}\left(T-T_{R E F}\right)\right] \underline{g} \\
& \frac{\partial T}{\partial t}+\underline{\nabla} \cdot[\underline{V T}]=\alpha_{0}^{\text {liquid }} \nabla^{2} T
\end{aligned}
$$

where $\rho_{0}=\rho\left(T_{R E F}\right), \beta_{T}=-\left.\frac{d \rho}{d T}\right|_{T_{R E F}}$ is generally known as the thermal expansion coefficient, $v=\mu / \rho$ is the kinematic viscosity and $\alpha$ is the thermal diffusivity defined as $\alpha=\lambda / \rho c_{p}\left(v_{0}\right.$ and $\alpha_{0}^{\text {liquid }}$ being the values of $v$ and $\alpha$ evaluated for $T=T_{\mathrm{REF}}$ ).

\subsubsection{Solid region}

In the solid region the energy equation reduces to the well-known heat diffusion equation, which assuming the properties of the solid (density, specific heat and thermal conductivity) to be constant, simply reads:

$$
\rho^{\text {solid }} c_{p}^{\text {solid }} \frac{\partial T}{\partial t}=\lambda^{\text {solid }} \underline{\nabla} \cdot[\underline{\nabla} T]=\lambda^{\text {solid }} \nabla^{2} T
$$

\section{$\underline{3.3 \text { Boundary conditions }}$}

\subsubsection{External boundary of the solid region}

On the external left and right sides of the configuration sketched in Fig. 1 the value of the temperature has been kept fixed, assuming the left being the hotter side $\left(T=T_{\text {hot }}\right)$ and the right the colder one ( $\left.T=T_{\text {cold }}\right)$. On the other walls, the so-called convective heat exchange boundary condition has been implemented:

$$
\left.\lambda^{\text {solid }} \frac{\partial T}{\partial n}\right|_{\text {wall }}=-h^{\text {air }}\left(T_{\text {amb }}-T_{\text {wall }}\right)
$$

where $T_{\text {wall }}$ is the temperature of the external boundary and $h^{\text {air }}$ is the convective heat transfer coefficient accounting for the heat exchange due to the natural convection taking place in the external environment (an estimation for the order of magnitude of this coefficient can be obtained using available engineering correlations, see, e.g., Rohsenow et al. [33]).

\subsubsection{Solid-fluid transition}

Where the solid and fluid regions are in contact the thermal boundary conditions reduce to: 


$$
\begin{aligned}
& \left.\lambda^{\text {solid }} \frac{\partial T}{\partial n}\right|_{\text {solid }}=\left.\lambda^{\text {liquid }} \frac{\partial T}{\partial n}\right|_{\text {liquid }} \\
& \left.T\right|_{\text {solid }}=\left.T\right|_{\text {liquid }}
\end{aligned}
$$

which express the continuity of heat flux and temperature, respectively (the reader being referred to Table II for relevant information about the physical properties of perspex).

Table II: Physical properties of perspex

\begin{tabular}{ccc}
\hline \hline $\begin{array}{c}\rho \\
{\left[K g / \mathrm{m}^{3}\right]}\end{array}$ & $\begin{array}{c}c_{p} \\
{[\mathrm{~J} /(\mathrm{Kg} K)]}\end{array}$ & $\lambda$ \\
1190 & 1459 & {$[\mathrm{~W} /(\mathrm{mK})]$} \\
\hline \hline
\end{tabular}

\subsection{Non-dimensional parameters and equations}

The number of parameters potentially affecting the dynamics can largely be reduced if the problem is put in non-dimensional form. Such procedure formally leads to the emergence of a set of 'characteristic' non-dimensional numbers, these being:

$\operatorname{Pr}=\frac{v_{0}}{\alpha_{0}^{\text {liquid }}} \quad$ (Prandtl number)

$$
R a=\frac{g \beta_{T} \Delta T L^{3}}{v_{0} \alpha_{0}^{\text {liquid }}} \quad \text { (Rayleigh number) }
$$

$B i=\frac{h^{\text {air }} L}{\lambda^{\text {solid }}} \quad$ (Biot number $)$

in the present work all the physical properties appearing in these numbers have been evaluated for $T_{R E F}=T_{\text {cold. }}$. Moreover, the $\Delta T$ appearing in the definition of the Rayleigh number corresponds to the difference between $T_{\text {hot }}$ and $T_{\text {cold }}$, i.e. the temperature actively controlled (kept constant) at the two opposing external sides of the solid shell.

Therefore, this $\Delta T$ is not representative of the jump of temperature $\Delta T_{\text {eff }}$ effectively established in the fluid (owing to the presence of a portion of solid material between the surface where the temperature is actively controlled and the internal liquid). Conveniently, we define $\Delta T_{\text {eff }}$ as the jump between the average temperatures on the internal walls corresponding to the externally heated and cooled surfaces, respectively. As the $\Delta T_{\text {eff }}$ defined in this way is only a fraction of the imposed 
temperature jump, the needs arises for a variant of the Rayleigh number based on it, which we introduce as $R a_{\text {eff }}=\frac{g \beta_{T} \Delta T_{e f f} L^{3}}{v_{0} \alpha_{0}^{\text {liquid }}}$ (obviously satisfying the inequality $R a_{\mathrm{eff}}<R a$ ).

Using $\alpha_{0}^{\text {liquid }} / L, \rho_{0}\left(\alpha_{0}^{\text {liquid }} / L\right)^{2}$ and $L^{2} / \alpha_{0}^{\text {liquid }}$ as reference velocity, pressure and time, respectively, defining the non-dimensional temperature as $\left(T-T_{\text {cold }}\right) / \Delta T$, and expressing the physical properties of the fluid as $\mu=\mu_{0} \mu^{*}(T), \lambda^{\text {liquid }}=\lambda_{0}^{\text {liquid }} \lambda^{*}(T)$ and $c_{p}=c_{p 0} c_{p}^{*}(T)$ (where the starred quantities are non-dimensional functions accounting for the dependence of these quantities on temperature), eqs. (2) and (3) can be rewritten as:

$$
\begin{aligned}
& \frac{\partial \underline{V}}{\partial \mathrm{t}}+\underline{\nabla} \cdot[\underline{V} \underline{V}]+\underline{\nabla} p=\operatorname{Pr} \underline{\nabla} \cdot\left[2 \mu^{*}(\underline{\nabla} \underline{V})^{s}\right]+\operatorname{Pr} \operatorname{RaT} i_{\underline{g}} \\
& \frac{\partial\left(c_{v}^{*} T\right)}{\partial t}+\underline{\nabla} \cdot\left[c_{v}^{*} \underline{V T}\right]=\underline{\nabla} \cdot\left[\lambda^{*} \underline{\nabla} T\right]
\end{aligned}
$$

which, for constant physical properties $\left(\mu^{*}(T)=1, \lambda^{*}(T)=1\right.$ and $\left.c_{p}^{*}(T)=c_{v}^{*}(T)=1\right)$ reduce to:

$$
\begin{aligned}
& \frac{\partial \underline{V}}{\partial \mathrm{t}}+\underline{\nabla} \cdot[\underline{V} \underline{V}]+\underline{\nabla} p=\operatorname{Pr} \nabla^{2} \underline{V}+\operatorname{Pr} \operatorname{RaT} \underline{i_{g}} \\
& \frac{\partial T}{\partial t}+\underline{\nabla} \cdot[\underline{V T}]=\nabla^{2} T
\end{aligned}
$$

Similarly, the heat transport equation in the solid and the related boundary conditions in nondimensional form read:

$$
\begin{aligned}
& \frac{\partial T}{\partial t}=\xi \nabla^{2} T \\
& \left.\frac{\partial T}{\partial n}\right|_{\text {external wall }}=-B i\left(T_{\text {amb }}-T_{\text {wall }}\right) \\
& \left.\chi \frac{\partial T}{\partial n}\right|_{\text {solid }}=\left.\lambda^{*} \frac{\partial T}{\partial n}\right|_{\text {liquid }} \\
& \left.T\right|_{\text {solid }}=\left.T\right|_{\text {liquid }}
\end{aligned}
$$

where $\xi=\alpha^{\text {solid }} / \alpha_{0}^{\text {liquid }}$ and $\chi=\lambda^{\text {solid }} / \lambda_{0}^{\text {liquid }}\left(\alpha^{\text {solid }}=\lambda^{\text {solid }} / \rho^{\text {solid }} c_{p}^{\text {solid }}\right)$. 


\subsection{The Numerical Method}

We have solved the Navier-Stokes equations using a technique pertaining to the category of the socalled projection methods. All these methods rely on a fundamental property of viscous fluids. Every viscous fluid evolving under the effect of a driving force (be it a volume or surface force) and interacting with solid walls must necessarily possess some vorticity. From a mathematical point of view this is translated into a non-zero value of the curl of the velocity field. This quantity, mathematically represented by the vector product of the nabla (gradient) operator and $\underline{V}$, does not depend directly on the gradient of pressure existing in the fluid, as witnessed by the related governing equations (where $\underline{\nabla} p$ does not appear, see, e.g., [34,35]).

These methods are therefore articulated into a 3-step algorithm building on the triadic relationship among velocity, vorticity and pressure. Initially, a first approximated version of the velocity field $\left(\underline{V}^{*}\right)$ is computed by integrating a simplified version of the momentum equation obtained by dropping the gradient of pressure;

$\frac{\partial \underline{V}^{*}}{\partial \mathrm{t}}+\underline{\nabla} \cdot[\underline{V} \underline{V}]=\operatorname{Pr} \underline{\nabla} \cdot\left[2 \mu^{*}(\underline{\nabla} \underline{V})^{s}\right]+\operatorname{Pr} \operatorname{RaT} \underline{i_{g}}$

Though this velocity is not the final one, it possesses the correct amount of vorticity since, as explained before, $\underline{\nabla} \wedge \underline{V}$ does not depend on $\underline{\nabla} p$.

As a second step, another of the fundamental properties of these flows is invoked to get a closure of the problem from a mathematical point of view. It is the incompressibility of the fluid, mathematically represented by $\underline{\nabla} \cdot \underline{V}=0$. By correcting $\underline{V}^{*}$ with the previously disregarded $\underline{\nabla} p$, the final velocity field can be expressed as

$\underline{V}=\underline{V^{*}}-\mathrm{C} \underline{\nabla p}$

where $C$ is a constant. By forcing this expression into $\underline{\nabla} \cdot \underline{V}=0$ (third step) and assuming $C=\Delta t$ (where $\Delta \mathrm{t}$ is the time integration step) one gets an extra equation to be used to determine the pressure:

$\nabla^{2} p=\frac{1}{\Delta t} \underline{\nabla} \cdot \underline{V^{*}}$

The final velocity field obtained in this way is physically correct and, at the same time, satisfies the theorem of the inverse calculus (see , e.g., Ladyzhenskaya [36]), which ensures it is 'fixed' from a mathematical point of view.

The simulations presented in the following section, in particular, have been carried out using OpenFoam, which relies on the Finite Volume Method (FVM). Two main families of algorithms based on the modus operandi described above (projection method) are available in OpenFoam: the 
SIMPLE (Semi-implicit Method for Pressure Linked Equations) scheme (see Patankar [37]) and the PISO (Pressure Implicit Split Operator), proposed by Issa [38] in its original form. The results of the present study have been obtained following the latter implementation as it suits better problems where a steady state may not exist. It uses a collocated grid approach, according to which all the variables are defined in the center of the cells. In order to avoid the unphysical uncoupling of velocity and pressure that would be induced by such an arrangement, a special interpolation of the velocity on the cell faces is required accordingly (Rhie e Chow [39]).

The velocity and temperature equations have been discretized using a second order central difference scheme for both convective term and diffusion. In order to solve the resulting system of algebraic equations, a Preconditioned Bi-Conjugate Gradient (PBiCG) with an Incomplete Lower Upper (DILU) pre-conditioner has been used for velocity and temperature in the predictor step, while for the solution of the elliptic equation for the pressure a Generalized Geometric-Algebraic Multi-Grid (GAMG) has been employed. Moreover, simulations have been conducted by setting 0.5 as maximum value of the Courant number and maximum $\Delta \mathrm{t}<4.464 \times 10^{-6}$ (corresponding to a dimensional time integration step $\cong 0.05 \mathrm{~s}$ ).

\section{$\underline{3.6 \text { Validation and Mesh independence study }}$}

This section is devoted to a discussion of the proper mesh size to be used for the simulation. In particular, this specific point is addressed on the basis of a parametric study relying on the evaluation of a sensitive quantity (namely, the maximum of the streamfunction). For simplicity, Hadley flow with perfectly adiabatic walls is considered. Moreover, the study is carried out in the frame of the Boussinesq approximation (eqs. (5)-(7)).

The parameters used for the Hadley flow are Prandtl number $\operatorname{Pr}=6.9$ and Rayleigh number $R a=1.77 \times 10^{7}$, corresponding to $\Delta T=20^{\circ} \mathrm{C}$, which is the value we have used for the experiments described in Sect. 4. Three (uniform) mesh resolutions have been considered: 60x60, 100x100, 150x150 (see Table III).

Table III: Maximum value of non-dimensional stream function as function of mesh size $(\mathrm{Pr}=6.9$, $\mathrm{Ra}=1.77 \times 10^{7}$ )

\begin{tabular}{ccc}
\hline \hline Mesh & $\psi_{\max }$ & Variation $[\%]$ \\
\hline \hline $60 \times 60$ & 42.345 & 3.18 \\
$100 \times 100$ & 41.041 & 1.41 \\
$150 \times 150$ & 40.469 & $/$ \\
\hline \hline
\end{tabular}

As made evident by this table, a uniform mesh with resolution 100x100 makes the relative variation of the maximum value taken by the streamfunction smaller than $2 \%$. Tables IV and V finally provide relevant information about the validation of the overall approach described in this section (through quantitative comparison with the 2D and 3D results by De Vahl Davis and Jones [40] and Bennet and Hsueh [41], respectively). 
Table IV: Validation (2D) - $R a=10^{6}, \operatorname{Pr}=0.71, \mathrm{~A}=1$ (Legend: $|\psi|_{\max }$ - Maximum absolute value of the stream function, $v_{\max }$ - Max vertical velocity component on a horizontal mid-plane, $x_{\max }$ Position of $v_{\max }, u_{\max }$ - Max horizontal velocity component on a vertical mid-plane, $\mathrm{y}_{\max }$ - Position of $u_{\max } . N u_{\text {hot }}$ - Average Nusselt number on the hot boundary. $N u_{\max }$,hot - Maximum Nusselt number on the hot boundary, $y_{N u, \max }$ - Position of $N u_{\max , h o t}, N u_{\min , h o t}$ - Minimum Nusselt number on the hot boundary, $y_{N u \text { min }}$ - Position of $N u_{\text {min, hot }}$ ).

\begin{tabular}{ccc}
\hline \hline Parameter & Present results & De Vahl Davis and Jones [40] \\
\hline$|\psi|_{\max }$ & 16.919 & 16.750 \\
$v_{\max }$ & 216.09 & 219.36 \\
$x_{\max }$ & 0.0368 & 0.0379 \\
$u_{\max }$ & 64.97 & 64.63 \\
$y_{\max }$ & 0.848 & 0.850 \\
$N u_{h o t}$ & 8.913 & 8.817 \\
$N u_{\max , h o t}$ & 18.193 & 17.925 \\
$y_{N u, \max }$ & 0.0368 & 0.0378 \\
$N u_{\min , h o t}$ & 0.970 & 0.989 \\
$y_{N u, \min }$ & 1 & 1 \\
\hline \hline
\end{tabular}

Table IV: 3D Validation (air flow in a differentially heated cubic cavity with four adiabatic walls)$R a=10^{6}, \operatorname{Pr}=0.71, u_{\max , m p}$ - Max horizontal velocity component on the $\mathrm{z}=0.5$ midplane and related $\left(x_{\max }, y_{\max }\right)$ coordinates, $v_{\max , m p}-$ Max horizontal velocity component on the $\mathrm{z}=0.5$ midplane and related $\left(x_{\max }, y_{\max }\right)$ coordinates, $N u_{\text {cold }}$ - Average Nusselt number on the cold boundary, $N u_{m p, c o l d}$ midplane Nusselt number (pertaining to a vertical averaging in the middle $[\mathrm{z}=0.5]$ of the cold wall).

\begin{tabular}{ccccccc}
\hline \hline & $u_{\max , m p}$ & $\left(x_{\max }, y_{\max }\right)$ & $v_{\max , m p}$ & $\left(x_{\max }, y_{\max }\right)$ & $\left|N u_{\text {mp,cold }}\right|$ & $\left|N u_{\text {cold }}\right|$ \\
\hline \hline Ref [42] & 70.91 & $(0.5000,0.1443)$ & 218.1 & $(0.0331,0.5000)$ & 9.012 & 8.77 \\
Ref [43] & 123.47 & $(0.1867,0.0634)$ & 218.25 & $(0.0362,0.4647)$ & 8.877 & 8.64 \\
Ref [44] & 68.34 & $(0.0005,0.1397)$ & 217.6 & $(0.0342,0.5000)$ & - & 8.6973 \\
$\begin{array}{c}\text { Ref [41] } \\
\text { Present }\end{array}$ & 122.8 & $(0.1920,0.0692)$ & 217.6 & $(0.0342,0.4500)$ & 8.875 & 8.64 \\
$\quad$ results & & & & & & \\
$\left(\right.$ grid 81 $\left.1^{3}\right)$ & 122.7 & $(0.1852,0.0617)$ & 215.1 & $(0.0370,0.5185)$ & 9.025 & 8.791 \\
\hline \hline
\end{tabular}

\section{Results}

The results section is articulated as follows. Section 4.1 is entirely dedicated to numerical simulations conducted under the constraint of two-dimensional (2D) flow using the model represented by eqs. (1-3) and eqs. (8-10). Though neglecting the finite size of the container along the $\mathrm{z}$ direction actually limits the translational relevance of such results to situations of practical interest, these findings are instrumental in revealing the role played by non-adiabatic horizontal 
boundaries and the cavity inclination in exciting specific convective modes and flow features (which would otherwise be absent). Among other things, these results also contribute to a topic of great theoretical (purely academic) interest, that is, the existence of 'multiple solutions' in these problems. Though this specific subject has attracted much attention in the case of fluids heated from below (Rayleigh-Bénard convection), recently some authors have shown that transition from the multiple solutions of the RB to the classical single roll configuration, which is characteristic of the Hadley flow, occurs through a series of extremely interesting collisions of bifurcation points (Torres et al. [45]; Wang et al. [46]).

The experimental results are presented in Sect. 4.2, where comparison with the idealized 2D simulations is stressed, pointing out analogies and differences, and success and limits of the 2D approach. Section 4.3 is finally used to justify the observed discrepancies between experiments and $2 \mathrm{D}$ computations on the basis of 'ad hoc' three-dimensional (3D) simulations. In that section the focus is on the counter-intuitive role played by non-adiabatic walls constraining the fluid along the third direction, which in place of contributing to the progression towards chaos have just the opposite effect.

As explained in Sect. 3, all cases presented here refer to water with variable properties encapsulated into a shell of solid perspex. In particular, first we examine circumstances where the prevailing temperature gradient is perfectly perpendicular to gravity (classical Hadley flow) for which the interpretation of the dynamics is relatively straightforward and then we move to the much more complex situation in which the direction of this gradient becomes inclined with respect to the horizontal direction. While for the former situation the flow is relatively stable in the considered range of parameters, the emerging patterns being limited to stationary or single-frequency states, in the latter progression towards fully developed chaos is possible. As already explained before, results are discussed following a peculiar order of presentation, which reflects our intention to implement a precise analysis hierarchy where the degree of complexity of the problem is progressively increased, i.e. 2D computations $\rightarrow$ Experiments $\rightarrow$ 3D simulations.

\subsection{Two-dimensional numerical simulations}

Following the approach outlined above, initially we explore the response of thermal convection induced in the considered configuration by a horizontal temperature gradient on the basis of purely 2D simulations.

The temperature difference $\Delta T$ is fixed to $20^{\circ} \mathrm{C}$, while three different values of $T_{\text {cold }}$ are considered for a fixed $T_{\mathrm{amb}}=22{ }^{\circ} \mathrm{C}$ (leading accordingly to three distinct average temperatures of the system represented by the fluid and the surrounding shell). The parametric investigation also involves an assessment of the heat exchange rate with the external environment properly quantified via the Biot number defined by eq. (13) $(B i=0.0526,0.2632$ and 0.5263 , which in dimensional terms would correspond, for a container having inner dimension $4 \mathrm{~cm}$, to values of convective coefficient $h=2$, 10 and $20 \mathrm{~W} / \mathrm{m}^{2} \mathrm{~K}$, respectively). 
Assuming three values of $T_{\text {cold }}\left(20,40,60{ }^{\circ} \mathrm{C}\right)$ and for each of them the three values of the Biot number indicated above leads to a matrix of 18 cases: 9 for the Hadley configuration and 9 for the inclined cavity. Related results are conveniently presented in terms of maps. These maps (see Fig. 5 for the case of horizontal temperature gradient) are organized in the following way: each row collects the cases with the same $T_{\text {cold }}$ while each column represents a specific value Biot number. Moreover each snapshot has been labelled as steady (S) or oscillatory (OS), and in the latter case (state following a Hopf bifurcation) the non-dimensional angular frequency of the oscillations $(\omega)$ has also been provided.
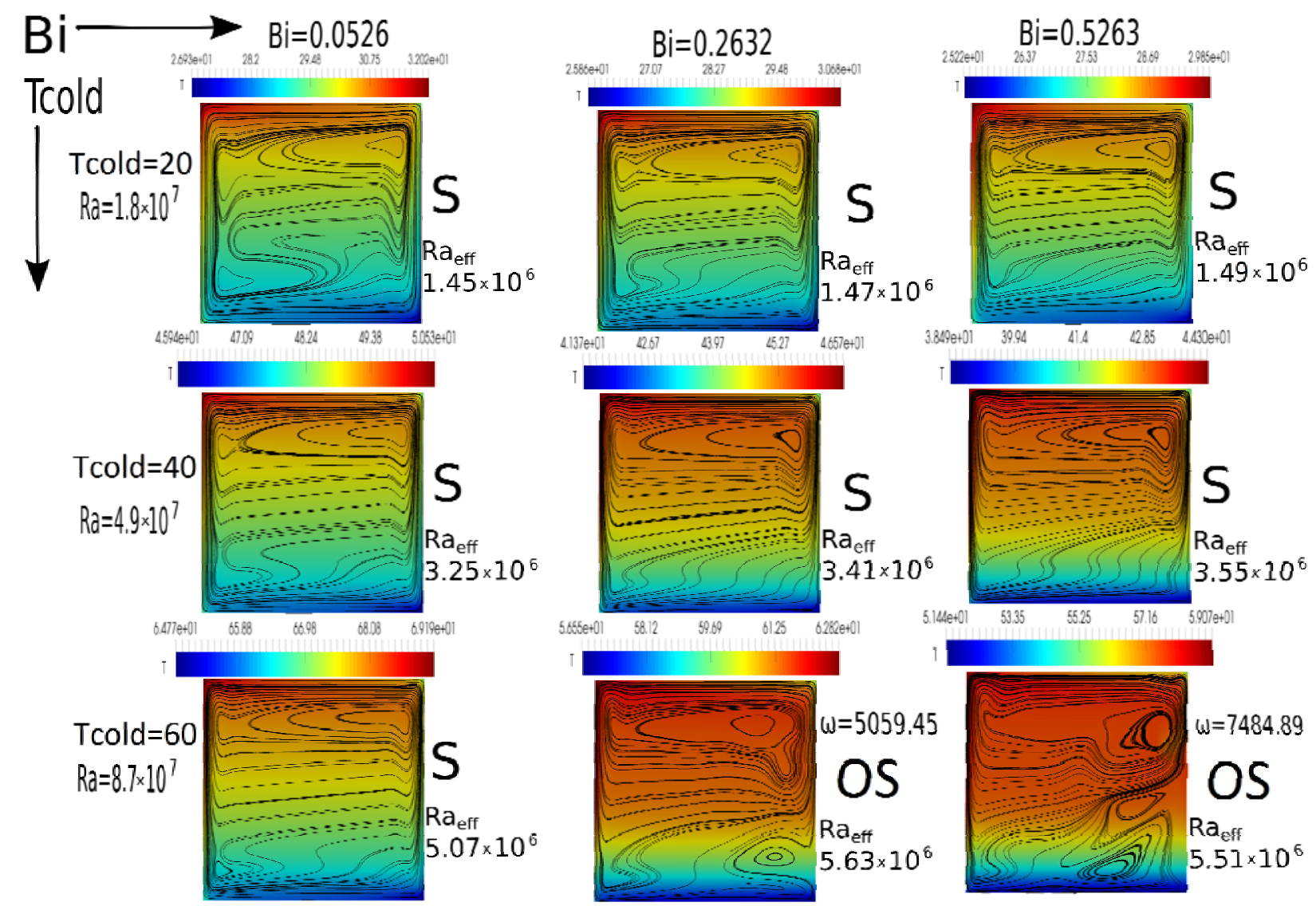

Figure 5: Map of possible spatio-temporal states (temperature and velocity distribution) as a function of $T_{\text {cold }}$ and $B i$ (temperature gradient perpendicular to gravity). $T_{\text {hot }}$ is on the left side of the cavity while $T_{\text {cold }}$ is on the right of the cavity. $\Delta T=20{ }^{\circ} \mathrm{C}$ for all considered cases (the changes in the Rayleigh number being due to a variation in the thermodynamic properties of the fluid). Legend: S (Steady state), OS (Oscillatory State, non-dimensional angular frequency $\omega$ evaluated using the reference time $\left.t_{\mathrm{REF}}=L^{2} / \alpha\right)$.

An example of the emerging oscillatory behaviors is reported in Fig. 6. In this figure (Hadley flow, i.e. $\Delta T$ perpendicular to $\mathrm{g}$ ) it is possible to notice that, as time passes, a strong roll located near the top right corner moves downwards, becoming weaker (frames 1, 2, 3) until a new roll nucleates above it and a new cycle of oscillation starts. 
These figures are instrumental in showing that, as expected, on increasing the Rayleigh number (for a fixed $\Delta T$, this is due to the variation induced in the physical properties of the fluid by a change of $\left.T_{\text {cold }}\right)$ the amount of vorticity being present in the physical domain grows appreciably. Moreover, following a typical behavior of the classical Hadley flow, a stagnant region is formed in the central region of the enclosure where fluid motion is essentially due to the entrainment of mass from the vertical boundary layers (confined in thin intruding flowing layers near the horizontal walls). When the steady unicellular Hadley convection enters such a boundary-layer regime, the temperature gradient in the core becomes small and a relatively stable central region emerges featuring a crossstream thermal stratification (many examples of this behavior being shown in Fig. 5).

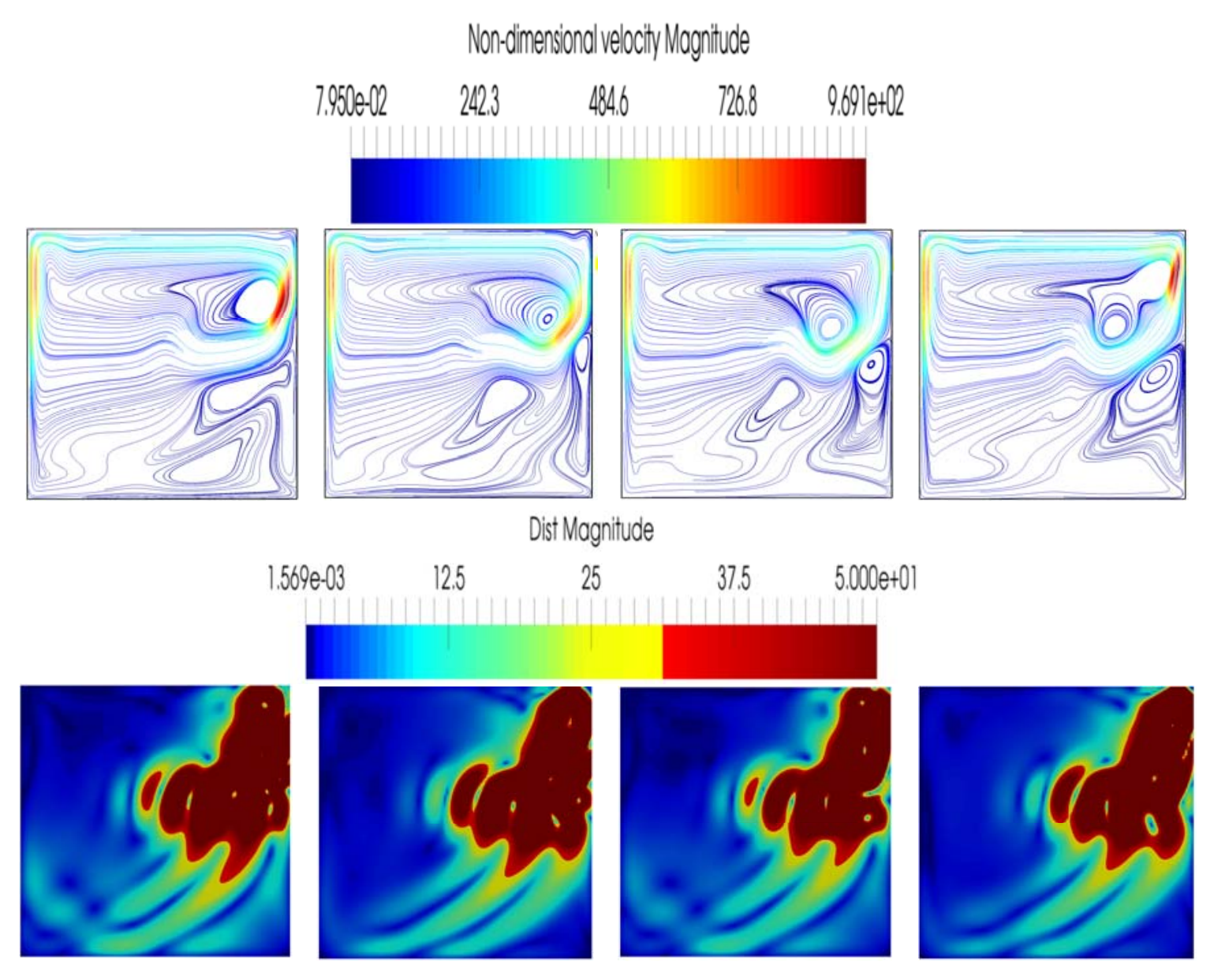

Figure 6: First row: Four snapshots of flow field evenly distributed along the period. First row Hadley flow with $T_{\text {cold }}=60^{\circ} \mathrm{C}$ and $B i=0.526, R a=8.7 \times 10^{7}\left(R a_{\mathrm{eff}}=5.51 \times 10^{6}\right)$. Second row: related disturbances.

In order to place the present results in a proper theoretical context, in the remainder of this section we will follow a specific approach, which heavily relies on the existing literature relating to buoyancy flow in enclosures with canonical (adiabatic or conducting) boundary conditions.

Meaningful cross-comparison with these classical situations will be used as a basis to interpret the present scenario and the related non-trivial impact of heat exchange with the external environment through the solid shell (in this regard, what follows should be regarded as a critical summary of 
potentially key aspects responsible for the dynamics observed in the present study, rather than a systematic and comprehensive survey of the existing literature).

In this context it is convenient to start from the simple remark that there is a long tradition of numerical studies dealing with the ability of buoyancy convection to produce permanent oscillatory states in differentially heated cavities. These analyses have revealed the incredible multiscale nature of the seemingly innocuous 2D problem even if idealized thermal boundary conditions are considered. As an example, for the case of adiabatic walls, the reader may consider the numerical simulations about square cavities filled with air performed by Le Quéré and Penot [47], Henkes and Hoogendoorn [48], Bucchignani [49], Yahata [50]. It is known that the flow can even develop turbulence (Paolucci and Chenoweth [51]; Paolucci [52]; Janssen and Henkes [53]; Xin and Le Quéré [54]; Farhangnia et al. [55]; Yahata [56]; Le Quéré and Behnia [57]; Mayne et al. [58]; Tian and Karayiannis [59]; Lappa [60]).

These instabilities have nothing to do with the typical perturbing mechanisms of the Hadley flow described in the introduction for $\operatorname{Pr}<\mathrm{O}(1)$ as the related hydrodynamic disturbances are not allowed for $\operatorname{Pr}>1$ (water). In practice, in this region of the space of parameters the aforementioned core region is known to remain stable and instabilities can only be produced by perturbations growing in the boundary layers located in proximity to the side-walls or similar effects.

Given these premises, relevant information for an initial interpretation of our results shown in Fig. 5 can be found in the study by Ravi et al. [61] for a square cavity yet in the case of air and adiabatic sidewalls. These authors were the first to highlight the emergence of a recirculating pocket of fluid close to the corners downstream of the vertical walls, and that the flow separates and reattaches along the horizontal walls in proximity to this recirculation (in conjunction with a significant increase in the thickness of the horizontal layer). Convincing arguments were provided that this corner structure is due to thermal effects (more precisely, due to the temperature undershoots in lateral boundary layer caused by the stable thermal stratification of the core, relatively cold fluid can reach the upper corner thereby causing the detachment of a cold plume from the ceiling, which in turn produces the observed separation and recirculation). Similar effects can be seen in Fig. 5.

Interestingly, Paolucci and Chenoweth [51] highlighted that oscillatory flow appearing in these circumstances is due to an instability that takes place near the aforementioned 'departing' corners in the detached flow region along the horizontal boundaries (occurring for $R a=\mathrm{O}\left(10^{8}\right)$ in a square cavity for Pr=0.71). Later, Le Quéré and Behnia [57] stressed that this mode breaks the usual centro-symmetry of the solution. They also provided an interesting interpretation for the underlying instability mechanisms, which they ascribed to the propagation of internal gravity waves excited by the peculiar structure of the detached flow region. Given the similarity in the patterning behavior (and with regard to the structure of disturbances reported in the second row of Fig. 6), we argue that flow detachment in proximity to the corner does still play a role in the present circumstances.

This argument, however, should not be taken as exhaustive. Indeed, we must keep in mind that, as the horizontal boundaries are not adiabatic, they might locally lead to the emergence of convective modes of RB nature. 
In this regard, once again critical comparison with the literature may help. In studies dealing with the classical case of conducting horizontal walls, the unstable stratification (and ensuing convective modes) close to the horizontal boundaries was identified as the reason for which the hierarchy of bifurcations relating to cavities with different thermal conditions can exhibit notable differences. As an example, for $\mathrm{Pr}=0.71$ the Hadley convection is known to become oscillatory in a square cavity for $R a=\mathrm{O}\left(10^{8}\right)$ and $R a=\mathrm{O}\left(10^{6}\right)$ for adiabatic and conducting boundaries, respectively (Jones and Briggs [62], the reader being also referred to Winters [63], Gelfgat and Tanasawa [64], and Xin and Le Quéré [65] for the specific configuration with conducting boundaries).

In the light of these results, with regard to the present results (Figs. 5 and 6), we come to the conclusion that the oscillatory convective structures visible in the lower part of the cavity must essentially be seen as localized Rayleigh modes, while those located in the upper part can be interpreted in the frame of the detachment flow widely discussed above. This interpretation is quantitatively substantiated by the disturbances reported in the second row of Fig. 6 (these disturbances have been obtained by subtracting the time-averaged velocity field to the instantaneous velocity). This figure is useful as it can be used to clearly discern the disturbances travelling in the vertical boundary layer close to the right wall (the large red spot clearly indicating the region where the flow undergoes detachment). It also clearly shows the presence of additional convective modes (with smaller magnitude) being located in the lower part of the cavity. Remarkably, in the present circumstances (where we take into account the effective heat exchange through the walls delimiting the fluid from above and from below), these two mechanisms combine (making the dynamics relatively complex, see also Lappa and Gradinscak [66]). The additional degree of freedom with respect to earlier studies conducted using the classical conducting walls (with temperature linearly varying form the cold side to the hot side) resides in the presence of horizontal walls with finite thickness and the effective distribution of temperature effectively established inside this wall, which in turn depends on the average temperature of the fluid and the intensity of the heat exchange with the external environment (as quantified by the Biot number). Indeed, this additional degree of freedom is at the root of the variety of cases reported in Fig. 5 as a function of $T_{\text {cold }}$ and $B i$.

The role of the Rayleigh mode becomes even more important when the inclined configuration is considered (Figs. 7 and 8). In particular, Fig. 8 illustrates the patterning behavior for $T_{\text {cold }}=60{ }^{\circ} \mathrm{C}$. As the reader will realize by inspecting this figure, the most evident feature in this case is the descending flow inclined with respect to the solid walls, displaying a variable intensity in time. The heat exchanged with the environment through the top wall can be so strong in some circumstances to produce a descending plume with ensuing emergence of two main (separated) circulation systems in the cavity.

Following up on the previous point, comparison of Fig. 8 with the map shown in Fig. 5 also makes evident the more chaotic nature of the patterns appearing in the former figure with respect to the steady one visible in the latter (inclining the cavity strongly contributes to the chaoticity of the emerging flow). 


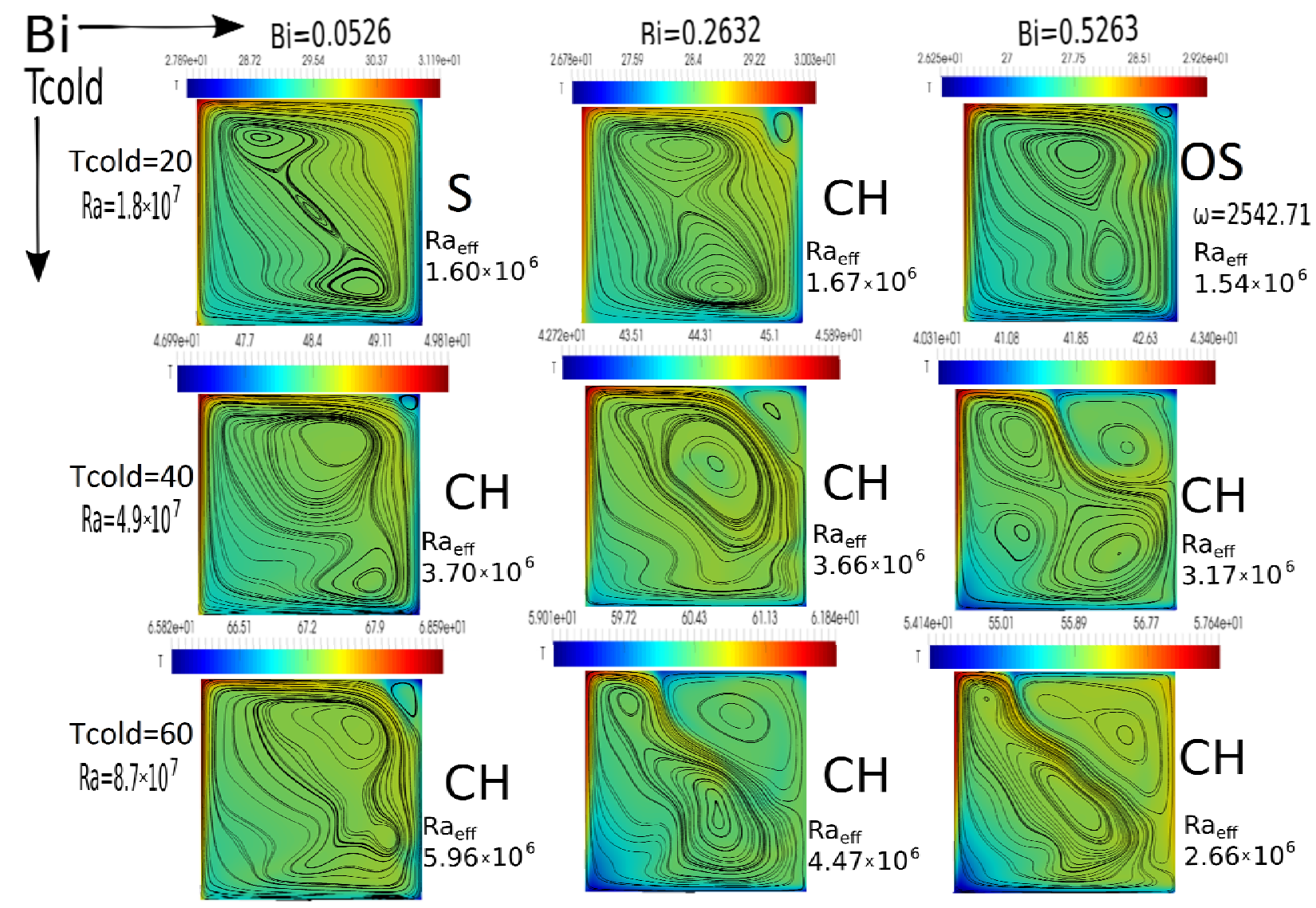

Figure 7: Map of possible spatio-temporal states (temperature and velocity distribution) as a function of $T_{\text {cold }}$ and $B i$ (temperature gradient inclined with respect to gravity, $\theta=40^{\circ}$ ). Legend: $\mathrm{S}$ (Steady state), OS (Oscillatory State), $\mathrm{CH}$ (Chaotic state).

Keeping all the concepts provided before in mind, the interpretation of our results for the case in which the heat exchange with the external environment is relatively weak (small values of $T_{\text {cold }} \cong T_{\mathrm{amb}}$ ) becomes straightforward. Taking a look, e.g., at Fig. 9 (corresponding to the frame located at the intersection of the first row and last column of Fig. 7) it is evident that the instability undergone by the system is a disturbance travelling along the boundary layer. Among other things, this finding is in qualitative agreement with the numerical study by Williamson et al. [67], who performed several simulations of a tilted square cavity with adiabatic top and bottom walls filled with a fluid having $\operatorname{Pr}=7$ (like that considered here), and found that the first instability which arises increasing the inclination (starting from an Hadley configuration) is yet located in the boundary layers (the interested reader may also consider the studies by Yang et al. [68,69], Lock and Han [70], Catton et al. [71], Kuyper et al. [72] and Adachi and Mizushima [73] for configurations with canonical boundary conditions). 


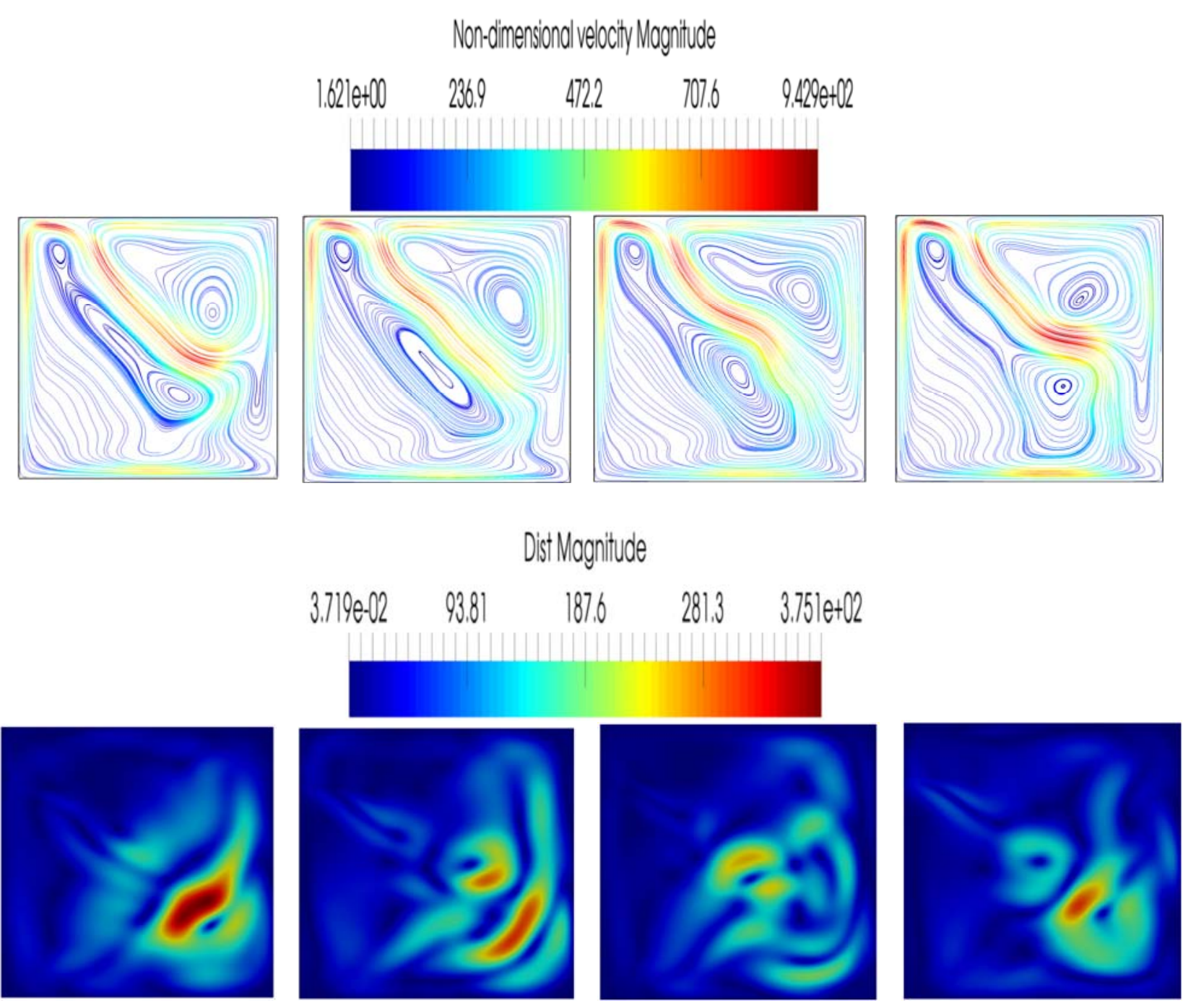

Figure 8: Four snapshots of the flow field evenly distributed along the main period (first row) and related disturbances (second row) for the case $T_{\text {cold }}=60{ }^{\circ} \mathrm{C}, \theta=40^{\circ}, B i=0.526, R a_{\mathrm{eff}}=2.66 \times 10^{6}$, $R a=8.7 \times 10^{7}$.

However, the situation becomes markedly different when the magnitude of the heat exchange increases. Indeed, comparison of Fig. $9\left(T_{\text {cold }}=20^{\circ} \mathrm{C}\right)$ with Figs. 8 and $10\left(T_{\text {cold }}=60{ }^{\circ} \mathrm{C}\right)$ makes clear that for higher $T_{\text {cold }}$ the unsteady behavior involves the internal part of the cavity as well.

Additional insights in this regard can be gathered from Fig. 11 where different frequency spectra relating to the solutions summarized in Fig. 7 have been collected. Though the spectra are essentially turbulent, it is possible to notice for $T_{\text {cold }}=60{ }^{\circ} \mathrm{C}$ and $B i=0.263$ (Fig. 11c) two main peaks of non-dimensional frequency: one located around $5 \times 10^{3}$, and another one around $10^{3}$. The first (higher amplitude) corresponds to a value of the frequency very similar to that found for the same conditions and no inclinations (see Fig. 5). Therefore, we argue that this specific peak corresponds to the boundary layer mode. 

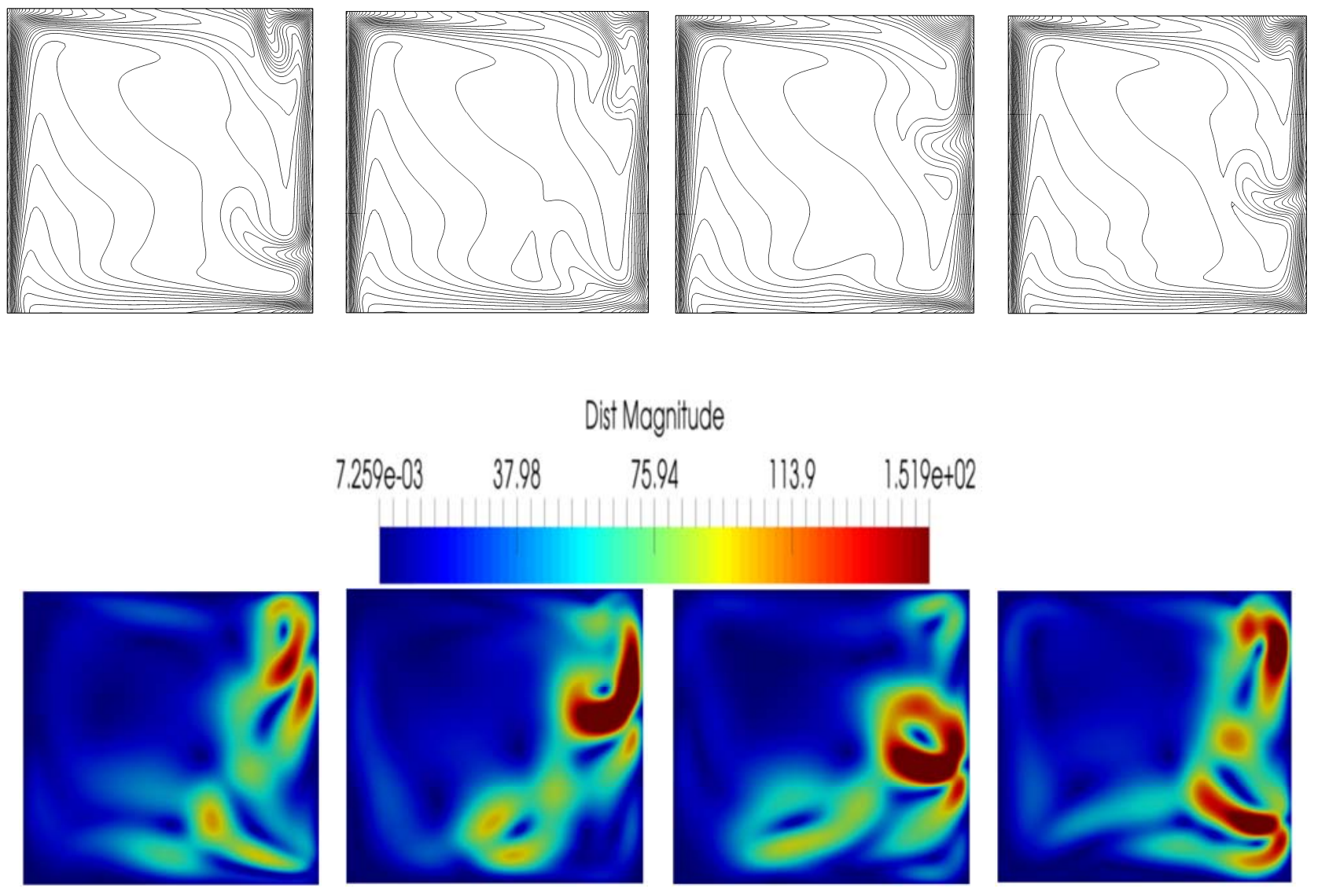

Figure 9: First row: Four snapshots of the temperature iso-lines evenly distributed along one period oscillation (first row) and related velocity disturbances (second row). $B i=0.526, T_{\text {cold }}=20{ }^{\circ} \mathrm{C}$, $\theta=40^{\circ}, R a=8.7 \times 10^{7}, R a_{\mathrm{eff}}=1.54 \times 10^{6}$. Second row: related velocity disturbances.
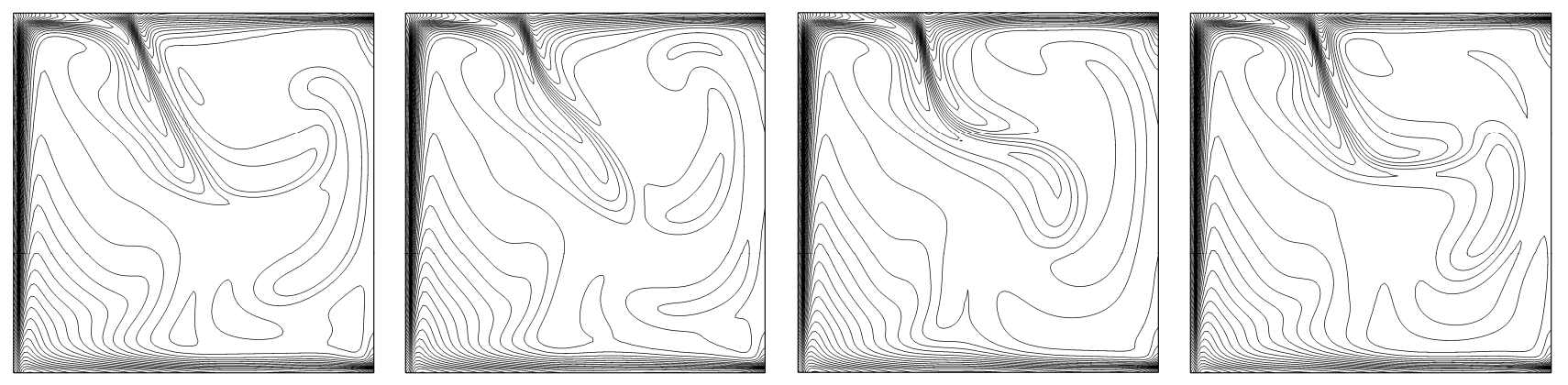

Figure 10: Four snapshots of the temperature iso-lines evenly distributed along the period. $B i$ $=0.263, T_{\text {cold }}=60^{\circ} \mathrm{C}, \theta=40^{\circ}, R a_{\text {eff }}=4.47 \times 10^{6}, R a=8.7 \times 10^{7}$. 


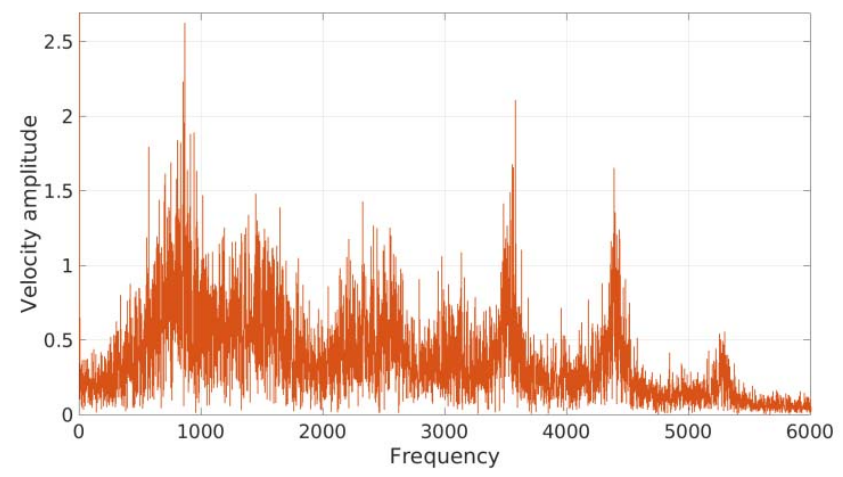

Fig. 11a: $T_{\text {cold }}=40{ }^{\circ} \mathrm{C}, B i=0.0526, R a=4.9 \times 10^{7}$

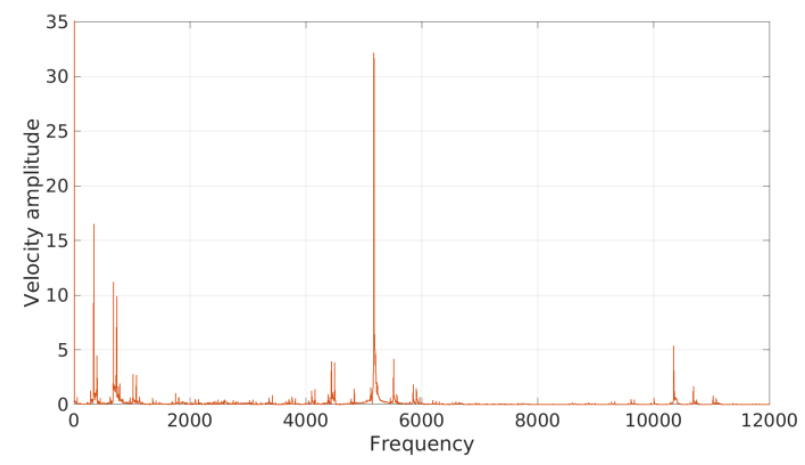

Fig. 11c: $T_{\text {cold }}=60^{\circ} \mathrm{C}, B i=0.263, R a=8.7 \times 10^{7}$

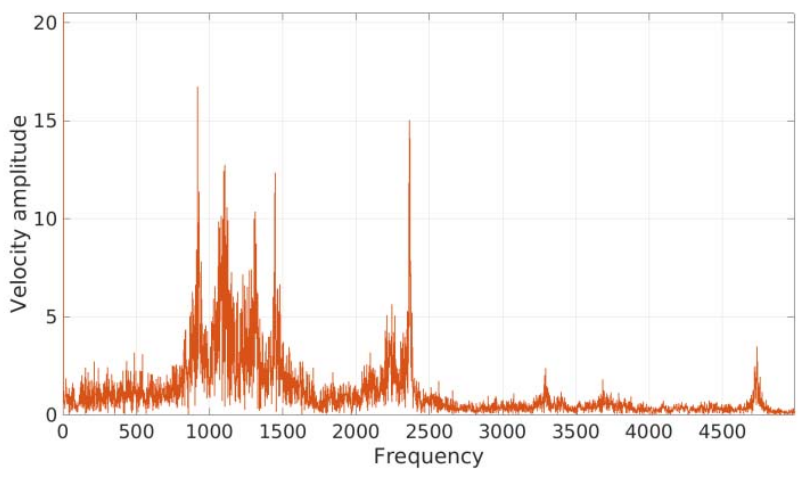

Fig. 11b: $T_{\text {cold }}=40{ }^{\circ} \mathrm{C}, B i=0.526, R a=4.9 \times 10^{7}$

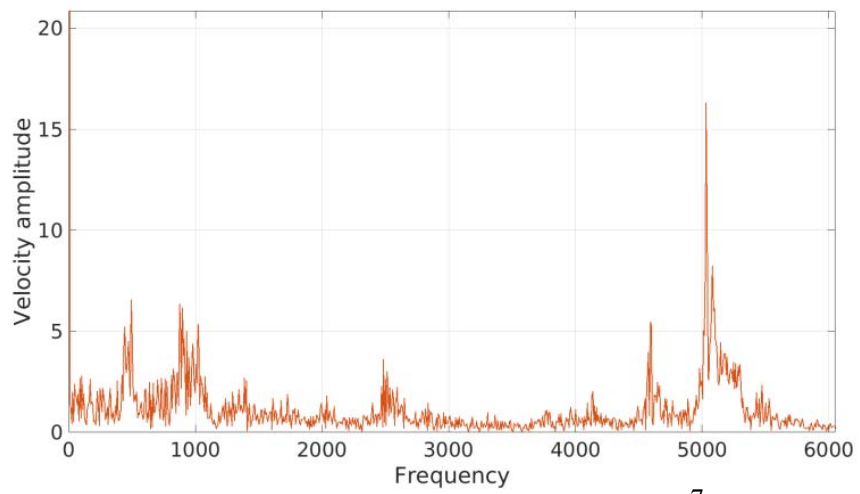

Fig. 11d: $T_{\text {cold }}=60^{\circ} \mathrm{C}, B i=0.526, R a=8.7 \times 10^{7}$

At $T_{\text {cold }}=40{ }^{\circ} \mathrm{C}$ (Fig. 11a and 11b), the lower frequency mode is dominant, which indicates a switch from the boundary-layer mode to a RB mode as dominant mechanism.

Non-dimensional velocity Manitude

$87.7560 .01 \quad 2555.4 \quad 510 \quad 764.5 \quad 1.0199+03$
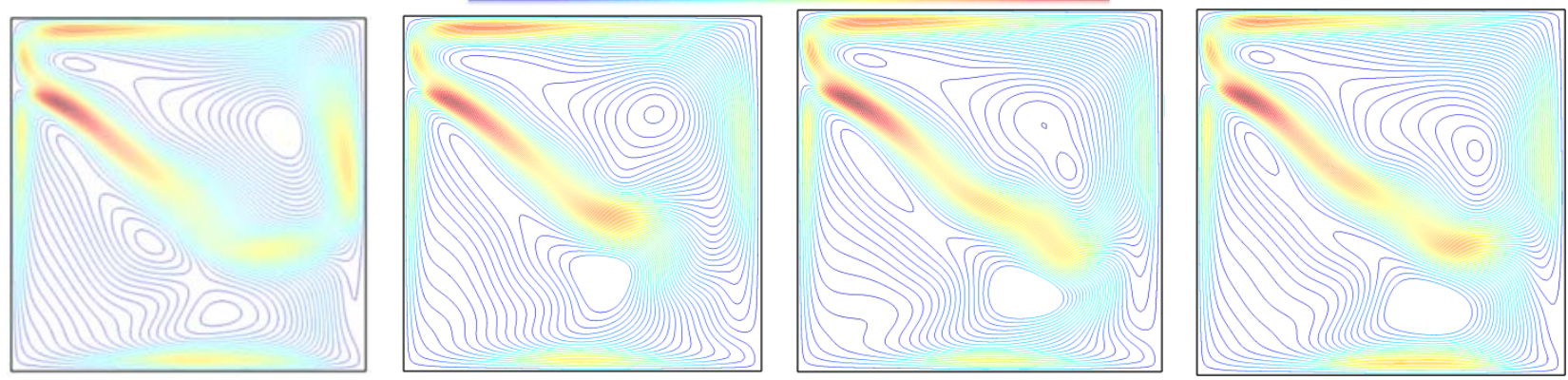

Figure 12: Four snapshots of the flow field evenly distributed along the main period of oscillation for $T_{\text {cold }}=60^{\circ} \mathrm{C}, B i=0.526, R a=8.7 \times 10^{7}$. 


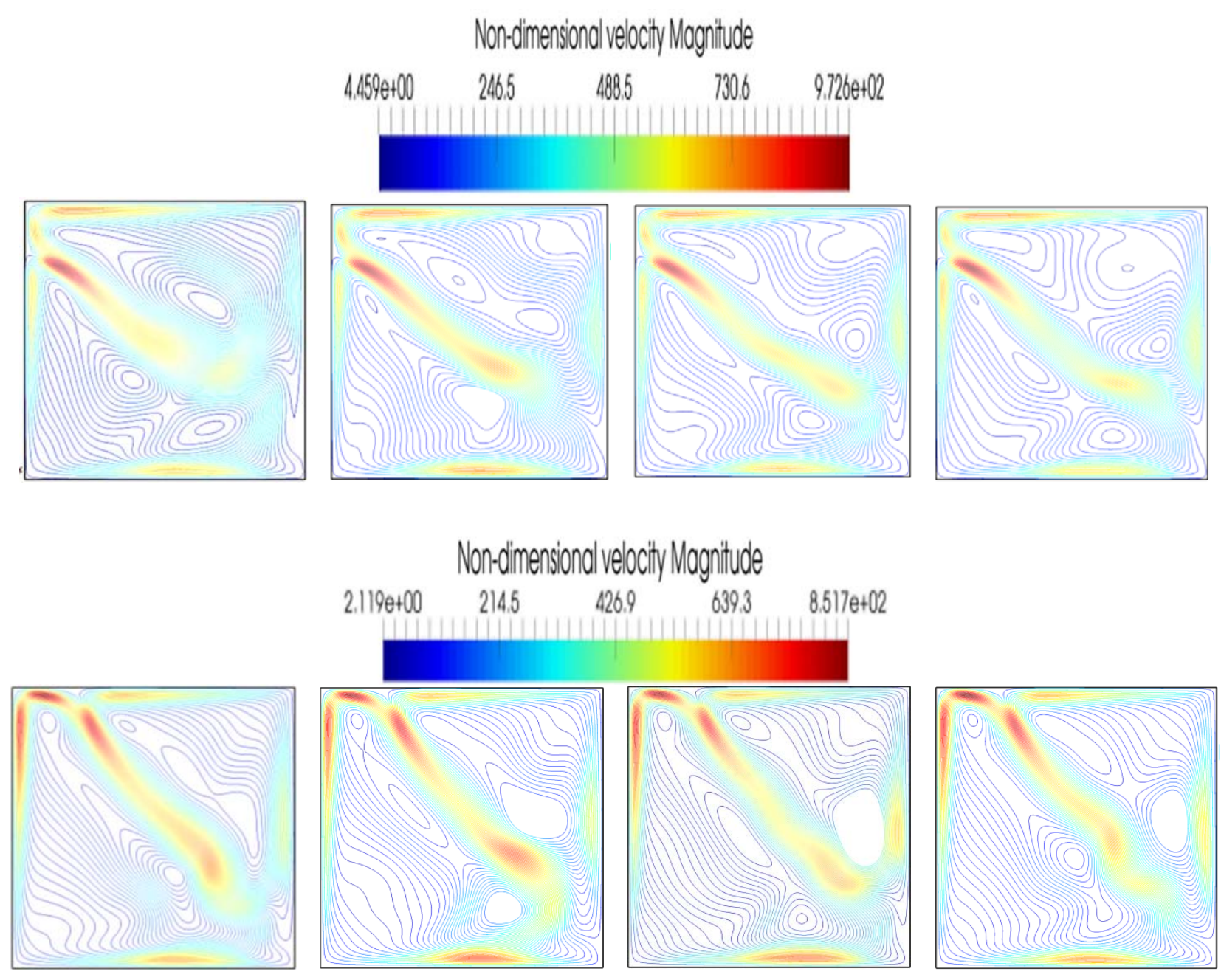

Figure 13: Multiple solutions (first and second row) for $T_{\text {cold }}=60^{\circ} \mathrm{C}, B i=0.79, R a=8.7 \times 10^{7}$.

Interestingly, in all these circumstances multiple solutions can also be produced (i.e. the flow can evolve towards different configurations for the same set of parameters when different initial conditions are considered), especially for relatively high values of $T_{\text {cold }}$ and $B i$.

In the present study we could find these solutions for specific values of $T_{\text {cold }}$ and $B i$, by exploring the system response when the basin of attraction is varied, i.e. by using the velocity and temperature fields found for other values of these parameters as initial conditions (see Lappa and Ferialdi [74-77] and references therein). A first example of such a peculiar behavior can be seen in Fig. 12 (to be compared with Fig. 8 for the same set of parameters, namely $T_{\text {cold }}=60{ }^{\circ} \mathrm{C}$ and $B i=0.526$ ).

Another example of multiple solutions is represented by Fig. 13. In Fig. 13a (first row) the plume is located on the hotter wall while in Fig. $13 b$ (second row) its position is on the top wall.

The interested reader may find other examples along these lines for standard thermal boundary conditions in Torres et al. [45]. These authors analyzed the transition in a cubic cavity from a Rayleigh-Bénard configuration to a one heated from the side at different Prandtl numbers focusing on the identification of the critical angles which lead the system from a state where multiple solution are possible (typical of the Rayleigh-Bénard convection) to the classical single roll 
configuration, which is characteristic of the Hadley flows. Similarly Wang et al. [78] demonstrated that the transition from the multiple solutions of the Rayleigh-Bénard to the single one of the system heated from the side occurs through a series of singularities and collisions of bifurcation points at specific critical angles. These extremely interesting studies were limited to adiabatic boundaries. The present results confirm that these initial-condition-dependent solutions can still be a feature of the system with more realistic boundary conditions considered here for a fixed inclination $\theta=40^{\circ}$, provided $T_{\text {cold }}$ and $B i$ are relatively high.

\subsection{Experimental results}

All the experiments (see Fig. 14) have been conducted using the facility and fluid containers described in Sect. 2 with an ambient temperature $\cong 22^{\circ} \mathrm{C}$.
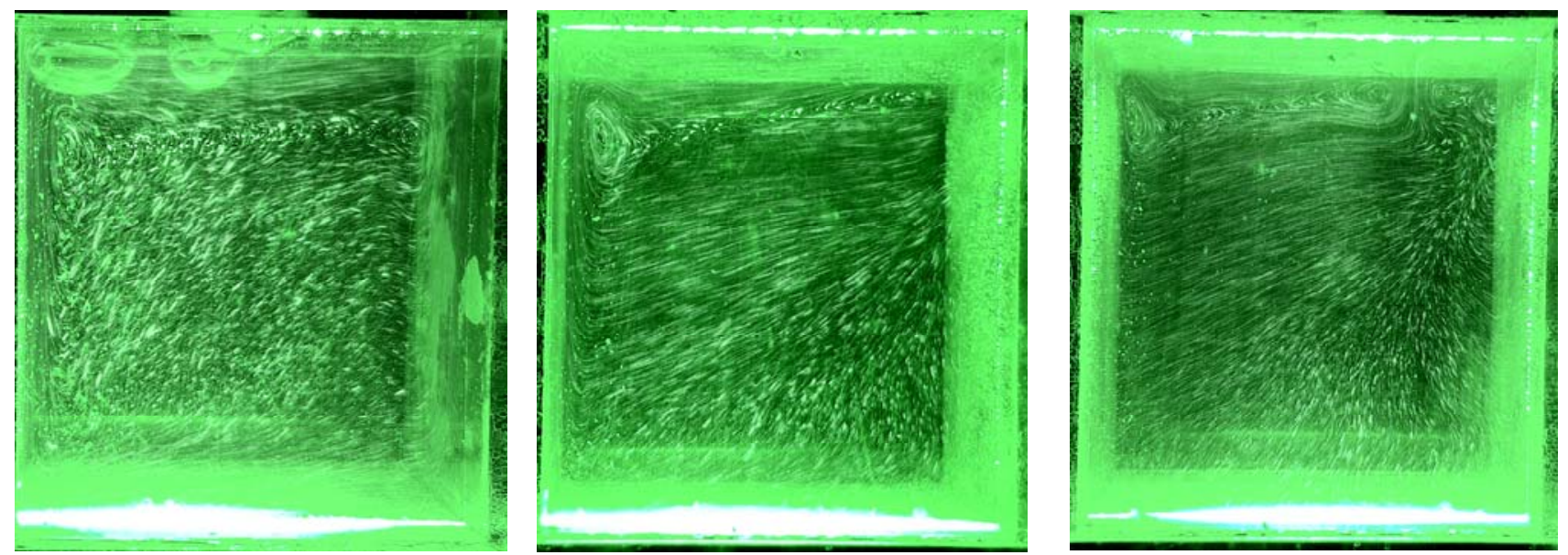

a)
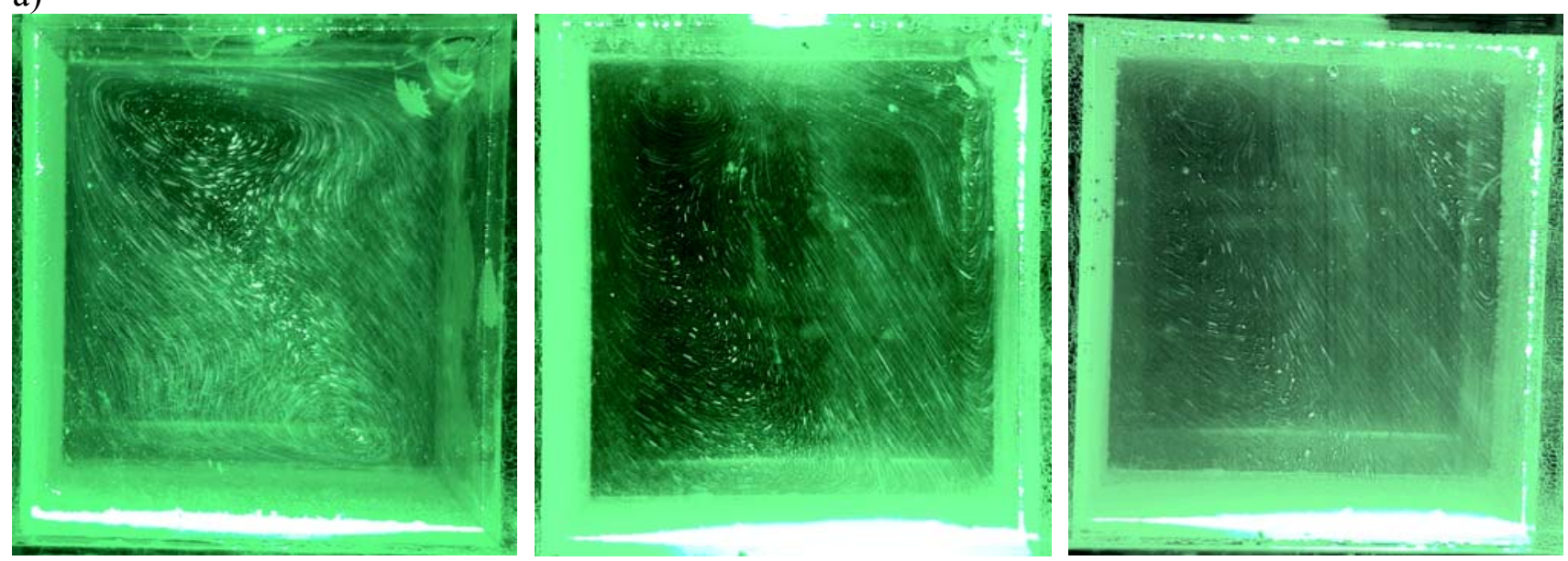

b)

Figure 14 : Stream -lines of six experimental cases $\left(\Delta T=20^{\circ} \mathrm{C}\right)$ : a) Hadley flow (starting from left) $T_{\text {cold }}=20^{\circ} \mathrm{C}, 40^{\circ} \mathrm{C}$ and $60^{\circ} \mathrm{C}$. b) $40^{\circ}$ inclined cavity (starting from left) $T_{\text {cold }}=20^{\circ} \mathrm{C}, 40^{\circ} \mathrm{C}, 60^{\circ} \mathrm{C}$. 
The experimental results collected in the first and second row of Fig. 14 (for the cases with horizontal and inclined temperature gradient, respectively) are quantitatively substantiated in Figs. 15 and 16. It can be seen that for fixed $\Delta T=20{ }^{\circ} \mathrm{C}$ (as in Sect. 4.1) and increasing values of $T_{\text {cold }}$ (20, 40 and $60^{\circ} \mathrm{C}$ in frames $\mathrm{a}, \mathrm{b}$ and $\mathrm{c}$, respectively), the flow is always steady.

In particular, the vector plot (shown on the left side) of any figure results from the application of a PIV (Particle Image Velocimetry) technique to experimental videos providing the evolution in time of tracer particles (size $50 \mu \mathrm{m}$ made visible by a light cut as shown in Fig. 14), while the corresponding horizontal velocity profile along a vertical line located at center of the cavity $(\mathrm{x}=0.02$ $\mathrm{m})$ is reported on the right of each figure.

We wish to highlight that the reconstruction of the velocity near the walls could not be very precise owing to the limited thickness of the boundary layers and the lack of sufficient resolution in these areas. Anyhow, the classical recirculation characteristic of the Hadley flows can be seen: the fluid rises near the hot side and sinks near the colder one. A roll is in proximity to the top left corner. Moreover, in the velocity profile the velocity peaks relating to the direct and return flow can clearly be identified (Fig. 15a).

Interestingly, the case with $T_{\text {cold }}=40{ }^{\circ} \mathrm{C}$ (Fig. 15b) shows the appearance of a localized circulation near the top right corner (colder side). Comparison with Fig. 15a reveals that the velocity peak corresponding to the return flow is replaced in these circumstances by an extended area of negative velocity, providing evidence for the existence of some 3D effects.

Moreover, for the maximum value of $T_{\text {cold }}\left(60^{\circ} \mathrm{C}\right)$, a well-defined counter-rotating roll (counterclockwise orientation) is established in proximity to the right upper corner (Fig. 15c). This circulation is driven by the strong heat exchange occurring through the upper wall with the external environment. As a result of heat loss, colder fluid produces a localized descending plume causing a departure from the classical Hadley flow, which would be attained in the case of adiabatic wall. The lack of a velocity peak close to the bottom wall provides clear hints about the presence of $3 \mathrm{D}$ effects also for this case.

As witnessed by Fig. 16, surprisingly, the flow is still steady when the inclined cavity $\left(\theta=40^{\circ}\right)$ is considered. However, the dynamics are qualitatively similar to those illustrated in Fig. 7: as $T_{\text {cold }}$ is increased with respect to the ambient temperature or $B i$ becomes higher, a circulation nucleates in the top right corner and grows in size. 

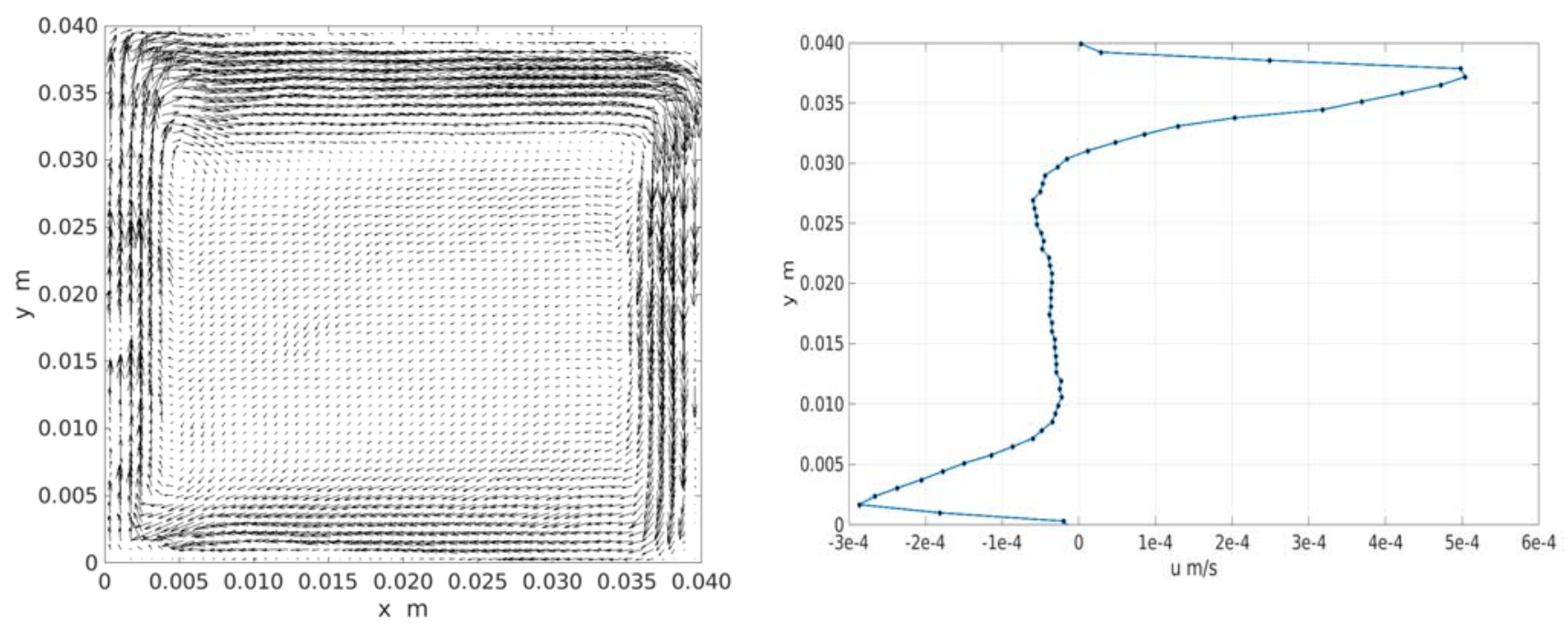

a)
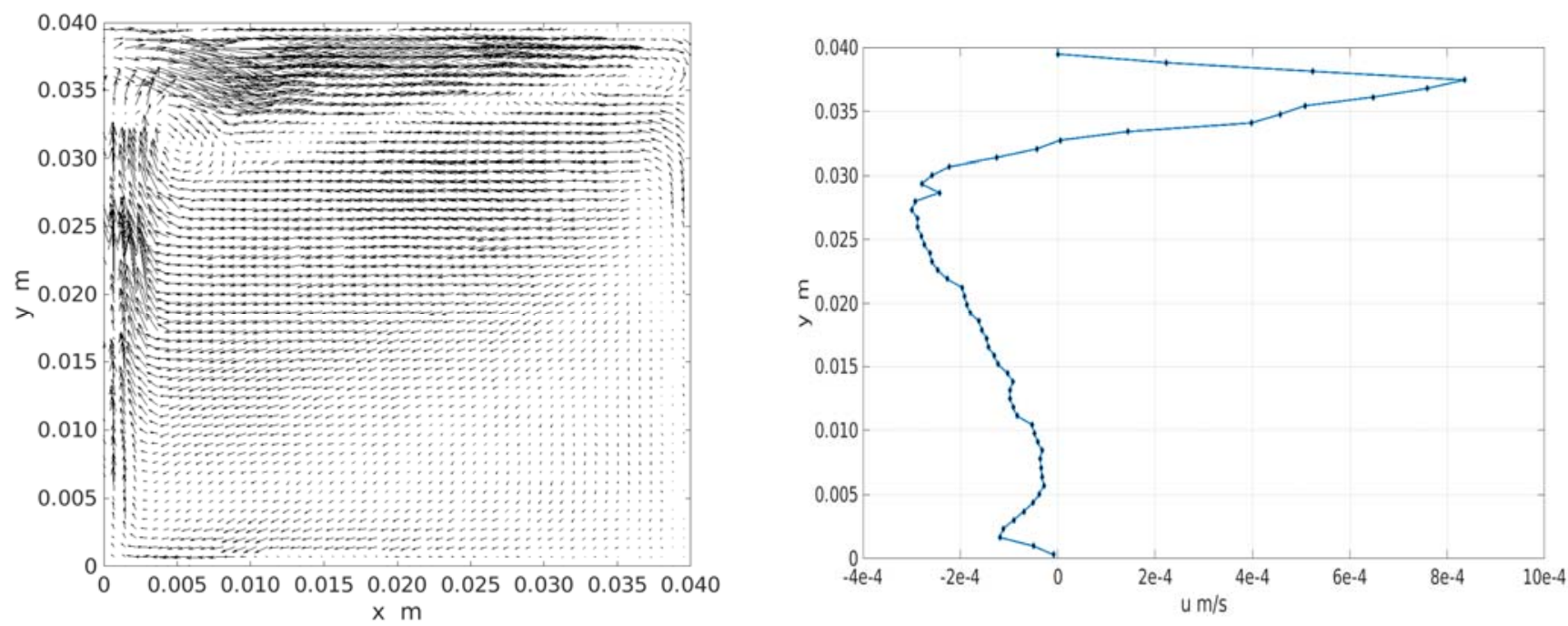

b)
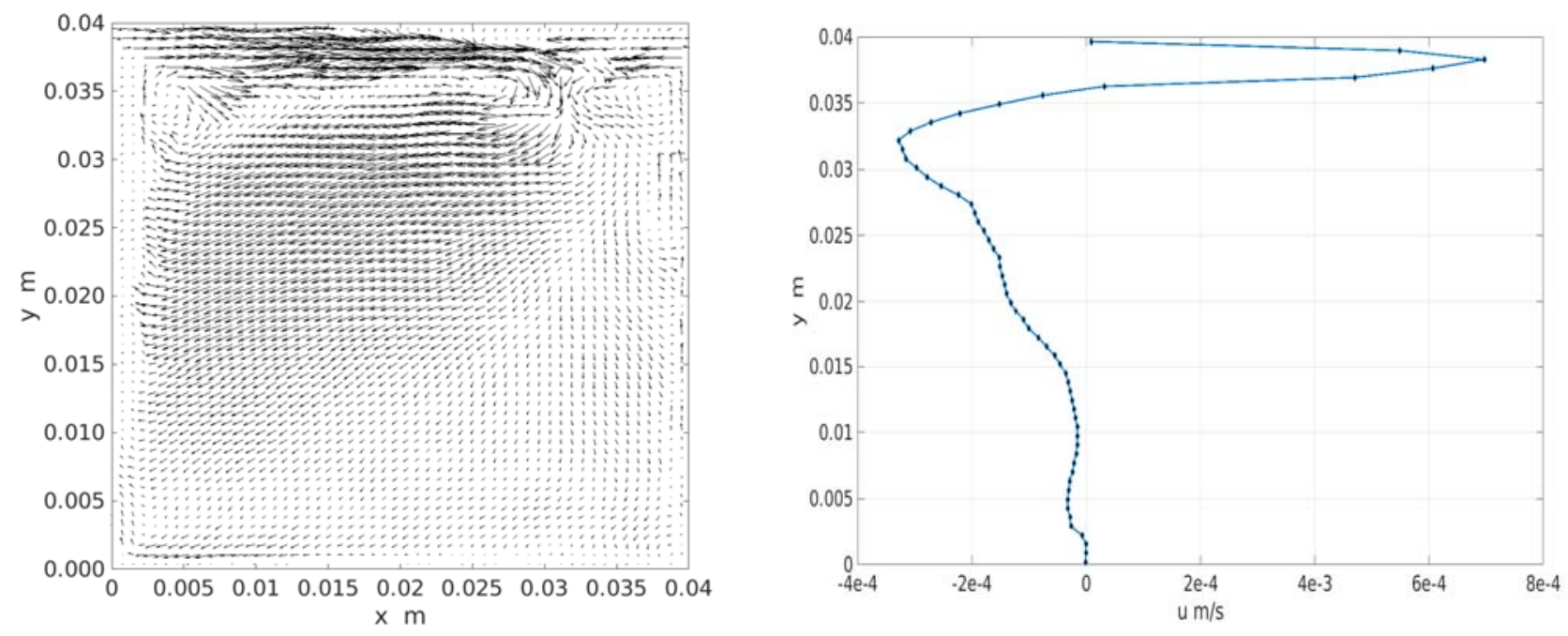

c)

Figure 15: Vector plot obtained with PIV technique for $\theta=90^{\circ}$ (hot side on the left, cold side on the right) and horizontal velocity component along a line at $\mathrm{x}=0.02 \mathrm{~m}$ (half of the cavity length): a) $T_{\text {cold }}$ $\left.=20^{\circ} \mathrm{C}, \mathrm{b}\right) T_{\text {cold }}=40^{\circ} \mathrm{C}$, c) $T_{\text {cold }}=60^{\circ} \mathrm{C}$. 

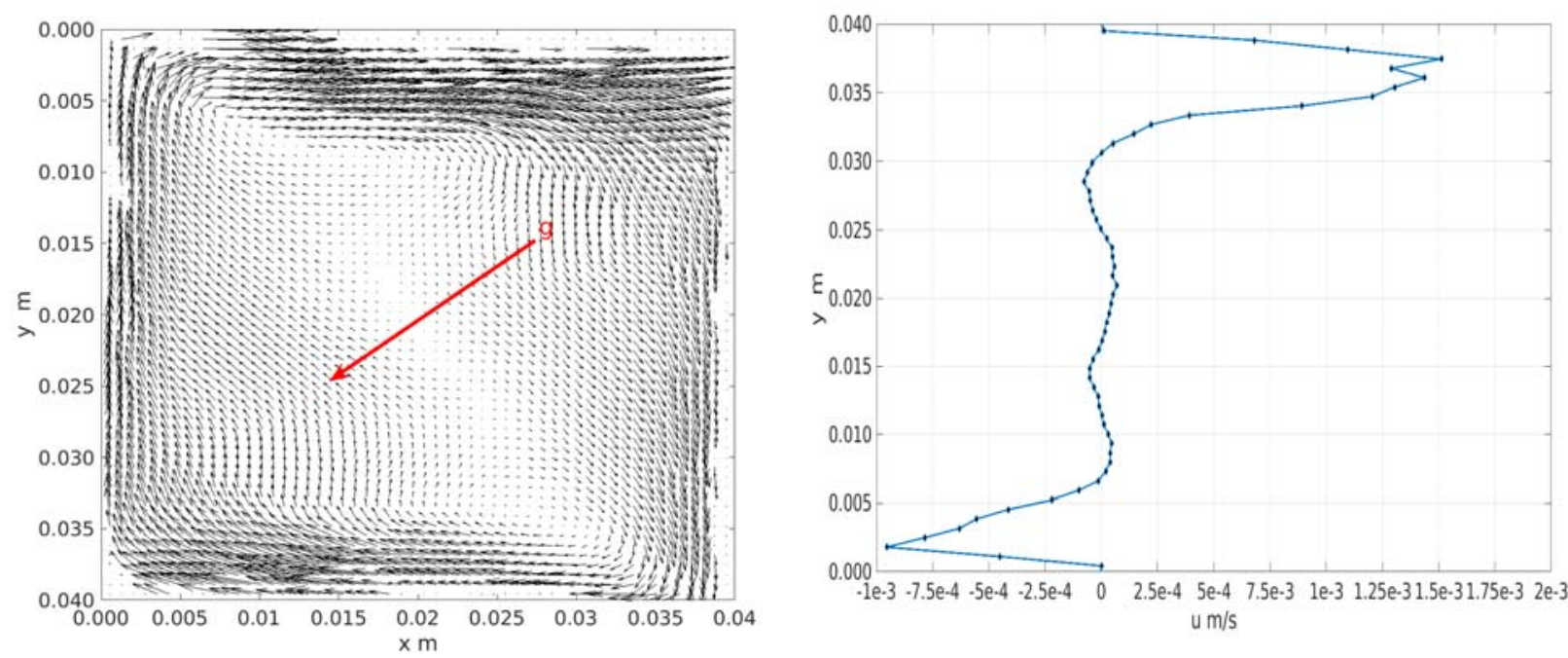

a)
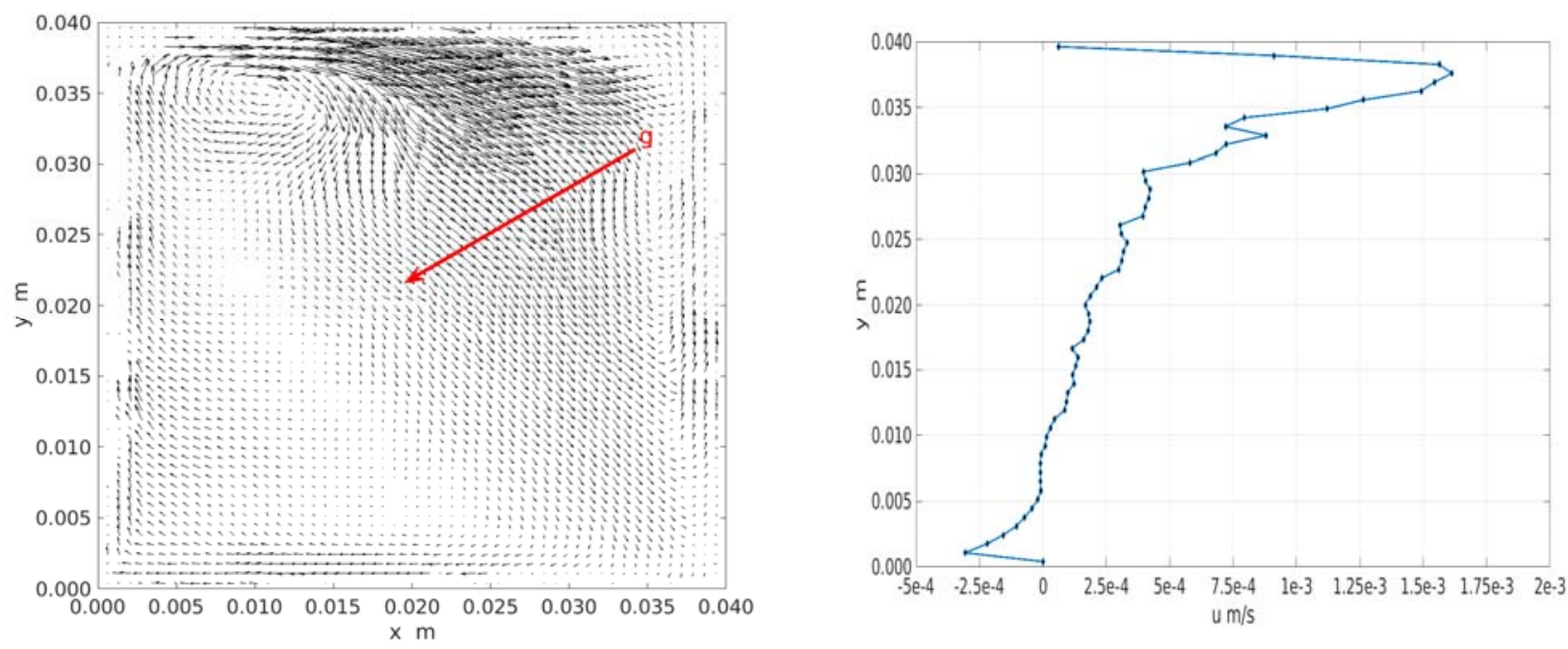

b)
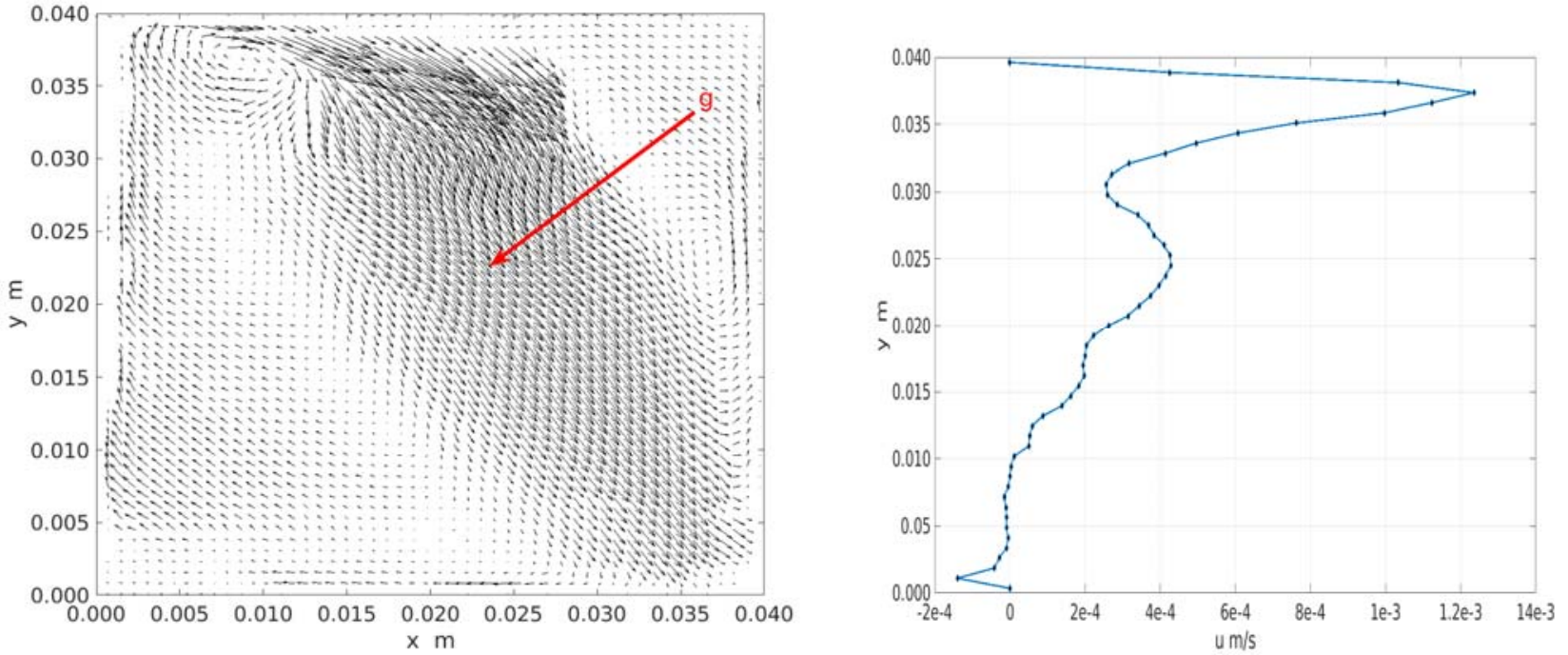

c)

Figure 16: Vector plot obtained with PIV technique for $\theta=40^{\circ}$ (hot side on the left, cold side on the right) and horizontal velocity component along a line at $\mathrm{x}=0.02 \mathrm{~m}$ (half of the cavity length): a) $T_{\text {cold }}$ $=20{ }^{\circ} \mathrm{C}$, b) $T_{\text {cold }}=40{ }^{\circ} \mathrm{C}$, c) $T_{\text {cold }}=60{ }^{\circ} \mathrm{C}$. 
Despite the qualitative similarities with the 2D computations, significant differences can be spotted with respect to the results presented in Sect. 4.1, which need to be properly interpreted and justified. Towards this end, dedicated 3D numerical results are presented in Sect. 4.3.

\subsection{Three-dimensional Numerical Simulations}

As illustrated at the end of the preceding section, the main difference between the present $2 \mathrm{D}$ results and the experiments relates essentially to the spatio-temporal nature of the flow. While only steady states have been obtained in the experiments (Fig. 14), the flow numerically simulated under the constraint of two-dimensionality can display a kaleidoscope of oscillatory, or even chaotic instabilities, especially when the heat exchange becomes more important (when $B i, T_{\text {cold }}$ or both are increased while keeping constant the ambient temperature $T_{\mathrm{amb}} \cong 22{ }^{\circ} \mathrm{C}$ ). This trend is particularly evident for the 2D inclined cavity (Fig. 7) and indirectly provides evidence that the heat exchanged by the system with the external environment through the walls delimiting it in the spanwise direction can have a significant stabilizing impact on the flow. This argument is further reinforced by the realization that the agreement between the $2 \mathrm{D}$ simulations and the experiments is relatively good only when the $T_{\text {cold }}$ is close to the ambient temperature and the Biot number is low. Indeed, for both Hadley and inclined configurations at $T_{\text {cold }}=20^{\circ} \mathrm{C}$ and $\mathrm{Bi}=0.0526$ (see the case corresponding to the first row and first column in Figs. 5 and 7), the computed pattern is steady and very similar to that found experimentally. This agreement progressively deteriorates as outlined above when the heat exchange becomes more important.
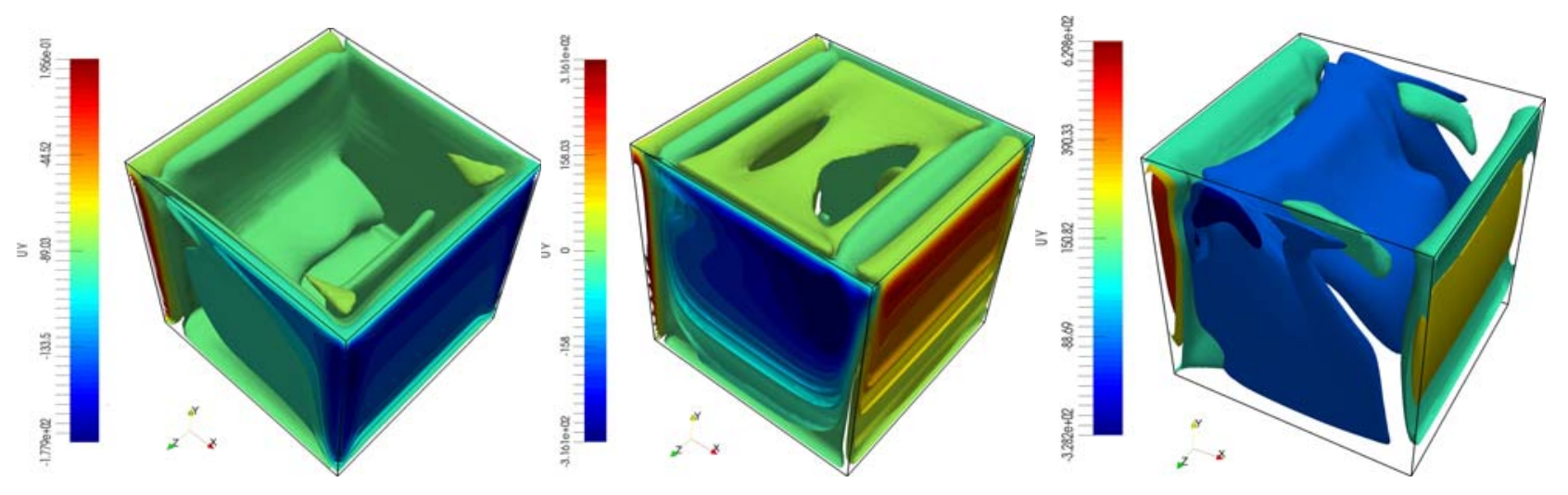

Figure 17: $3 \mathrm{D}$ simulations (mesh $\left.100^{3}\right)$ - Iso-surfaces of vertical velocity component $\left(\Delta T=20^{\circ} \mathrm{C}\right)$ : (a) Hadley flow: $T_{\text {cold }}=20^{\circ} \mathrm{C}, B i=0.0526, R a=1.8 \times 10^{7}, R a_{\text {eff }}=9.00 \times 10^{5}$; (b) Hadley flow $\left(\theta=90^{\circ}\right): T_{\text {cold }}$ $=60{ }^{\circ} \mathrm{C}, R a=8.7 \times 10^{7}, R a_{\mathrm{eff}}=4.63 \times 10^{6}, B i=0.526$; (c) Inclined cavity $\left(\theta=40^{\circ}\right): T_{\text {cold }}=60{ }^{\circ} \mathrm{C}$, $B i=0.526, R a=8.7 \times 10^{7}, R a_{\mathrm{eff}}=1.490 \times 10^{5}$. 

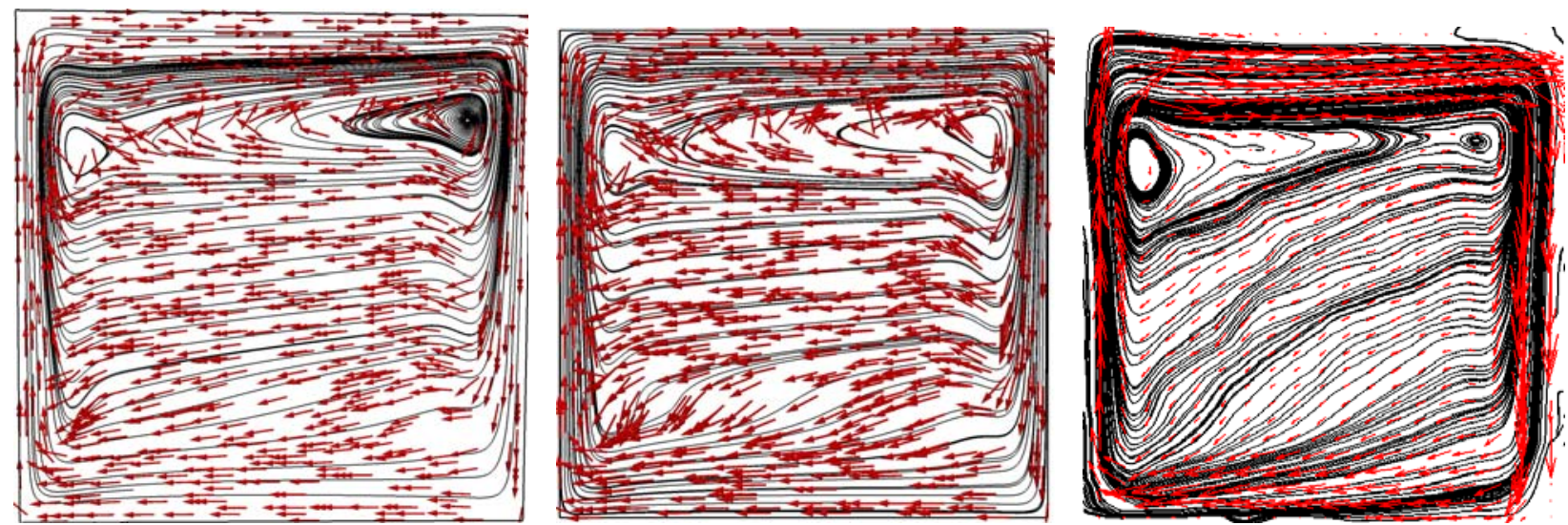

a) Case $\theta=90^{\circ}, \quad T_{\text {cold }}=20^{\circ} \mathrm{C}, \Delta T=20^{\circ} \mathrm{C}$ : Left - $2 \mathrm{D}$ simulation, $B i=0.0526, R a=1.8 \times 10^{7}$, $R a_{\mathrm{eff}}=1.45 \times 10^{6}$; Center - 3D simulation: $B i=0.0526, R a=1.8 \times 10^{7}, R a_{\mathrm{eff}}=9.00 \times 10^{5}$; Right Experiment.
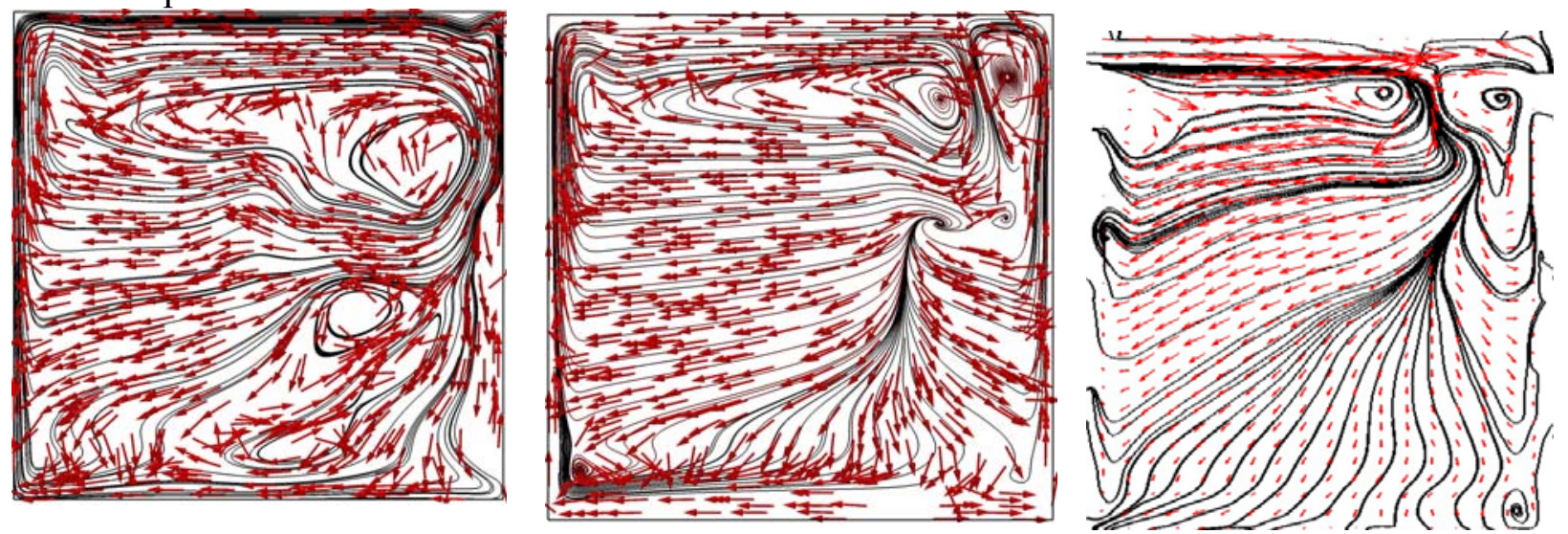

b) Case $\theta=90^{\circ}, \quad T_{\text {cold }}=60^{\circ} \mathrm{C}, \Delta T=20^{\circ} \mathrm{C}$ : Left - $2 \mathrm{D}$ simulation, $B i=0.526, R a=8.7 \times 10^{7}$, $R a_{\mathrm{eff}}=5.51 \times 10^{6}$; Center - 3D simulation: $B i=0.526, R a=8.7 \times 10^{7}, R a_{\mathrm{eff}}=4.63 \times 10^{6}$; Right Experiment.
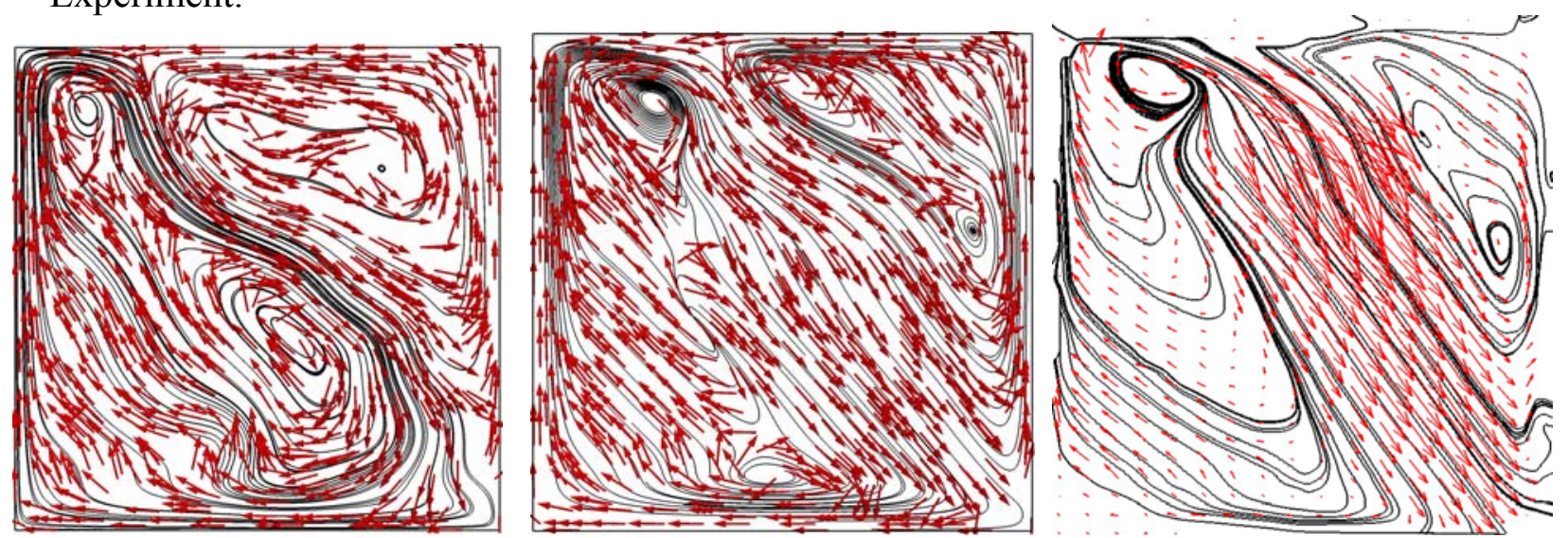

c) Case $\theta=40^{\circ}, \quad T_{\text {cold }}=60^{\circ} \mathrm{C}, \Delta T=20^{\circ} \mathrm{C}$ : Left - 2D simulation, $B i=0.526, R a=8.7 \times 10^{7}$, $R a_{\mathrm{eff}}=2.66 \times 10^{6}$; Center - 3D simulation, $B i=0.526, R a=8.7 \times 10^{7}, R a_{\mathrm{eff}}=1.490 \times 10^{5}$; Right Experiment.

Figure 18: Snapshots of velocity field: Comparison between 2D simulations, 3D simulations and experiments. 
At this stage, by presenting the 3D simulations, our aim is to produce more precise information to interpret the dynamics revealed by the experiments. Along these lines, Fig. 17 illustrates three different configurations: Hadley flow $\left(\theta=90^{\circ}\right)$ for $B i=0.0526, T_{\text {cold }}=20$ and $B i=0.526$ and $T_{\text {cold }}=60$ ${ }^{\circ} \mathrm{C}$ and inclined cavity $\left(\theta=40^{\circ}\right)$ at $T_{\text {cold }}=60^{\circ} \mathrm{C}, B i=0.526$. Additional insights can be gathered from Tables VI-XI and Fig. 18, where these three cases are compared reporting 2D, experimental and 3D results in a slice through the flow field (the xy mid-section).

In such a context, Fig. $18 \mathrm{a}\left(T_{\text {cold }}=20{ }^{\circ} \mathrm{C}\right)$ is particularly meaningful. What sets this specific behavior apart from the others shown in Fig. $18 \mathrm{~b}$ and $18 \mathrm{c}$ is that there is almost no difference between 2D and 3D numerical simulations and both predict pretty well the experimental outcomes in terms of spatio-temporal behavior (steady flow) and pattern (except for the precise size of the right top corner).

The good agreement between two-dimensional simulations and experiments is no longer there for the other two cases. As anticipated, the first and most remarkable feature, which makes a notable difference is the change induced in the nature of the flow by the dimensionality of the problem: it is oscillatory in the 2D numerical simulation while both experiments and the three-dimensional simulations are steady. This clearly indicates that accounting for the effective (finite) extension of the cavity along the spanwise direction can make the numerical results closer to the experimental outcomes. As evident in Fig. 18b, the counter-rotating roll in the top right of the cavity, which is not present when the domain is considered two-dimensional, is correctly captured by the 3D simulations. There are some differences between the 3D simulations and experiments, which we ascribe to the value of the Biot number used for the numerical simulations (based on estimates, which may not reproduce exactly reality).

In such a context, the flow structures collected in Fig. 17, where it can be seen that the velocity variations along the spanwise direction are relatively small and concentrated in proximity to the walls, provide strong hints that the mechanism for the departure of $3 \mathrm{D}$ results from the $2 \mathrm{D}$ simulations sensitively depends on purely thermal effects, i.e. the heat loss taking place through the walls along $\mathrm{z}$ (while the impact of frictional effects can be considered quite limited).

Unfortunately, comparison with the literature in these cases is not possible as most of existing numerical studies have been devoted to differentially heated 3D cavities with adiabatic walls (Schladow et al. [79], Kowalewski [80] and Melnikov and Shevtsova [1]; Bennett and Hsueh [41]; Lee and Lin [81]) for which the 3D effects simply consist of weak spiraling motions visible in the velocity field. For the Hadley flow in cubic cavities, Belmonte et al. [82] and Fusegi et al. [83] could experimentally get oscillatory flow (by increasing the applied temperature difference) due to the excitation of boundary-layer disturbances.

As a concluding remark for this study, we limit ourselves to pointing out that a further increase in the applied temperature difference (not possible with the present experimental apparatus, but affordable in terms of numerical simulation) would lead to oscillatory flow also for the 3D cases considered here. This is clearly shown in Fig. 19, where we have also reported the signal of velocity for the inclined cavity with $B i=0.526$ and a $\Delta T$ doubled with respect to that considered for all the 
other cases. This indicates that the time-dependent behaviors revealed by the simulations conducted under the unphysical constraint of 2D flow are not suppressed at all; rather they are shifted towards higher values of the Rayleigh number when realistic 3D configurations are considered.
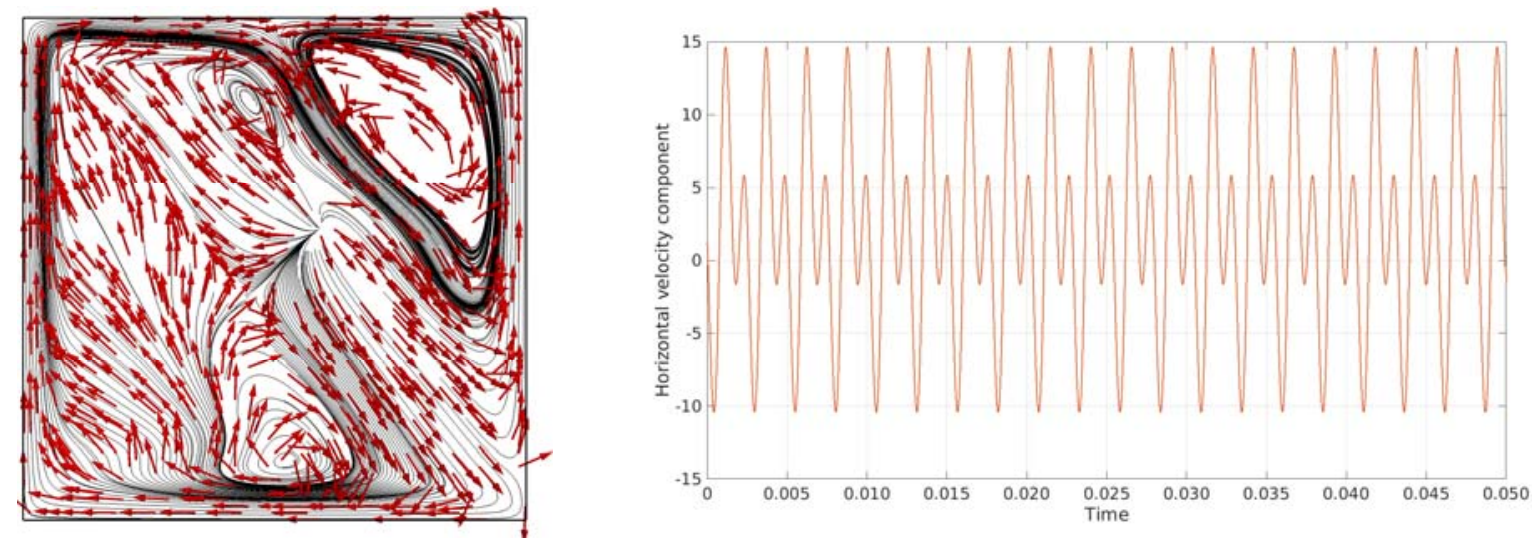

Figure 19: Snapshots of velocity field (left) and signal of velocity at a fixed point: $\theta=40^{\circ}$, $T_{\text {cold }}=60^{\circ} \mathrm{C}, \Delta T=40^{\circ} \mathrm{C}, B i=0.526$, non-dimensional angular frequency $\omega=2.47 \times 10^{3}$.

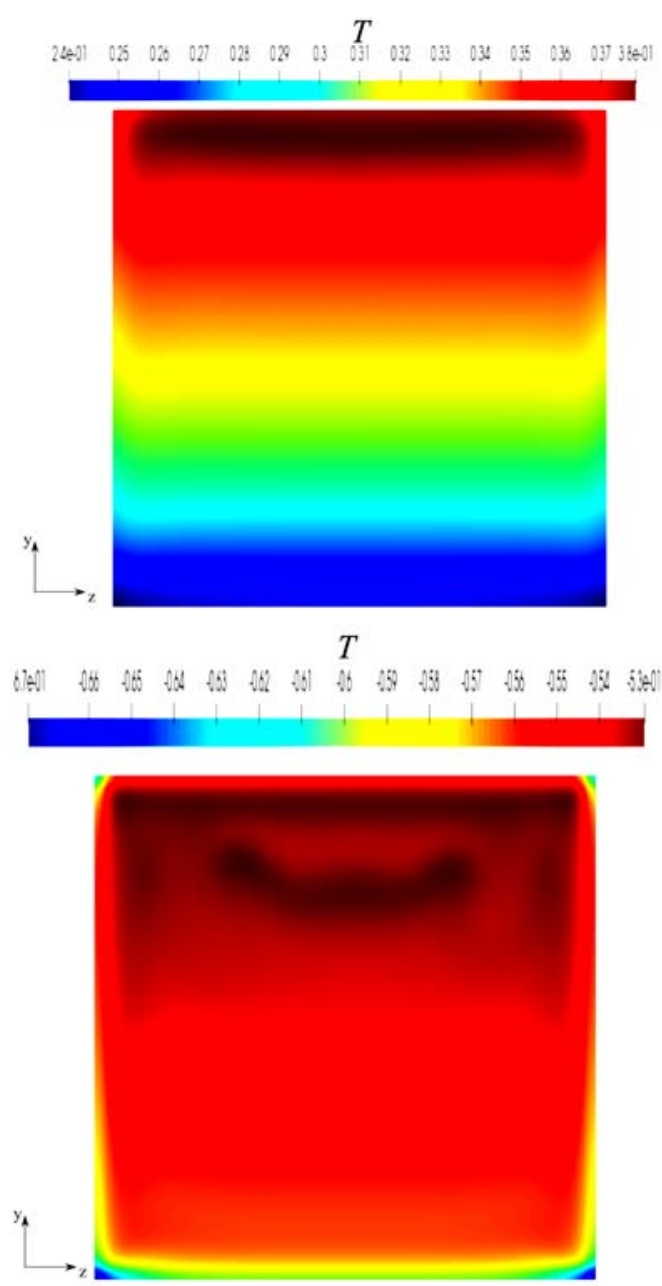

a)
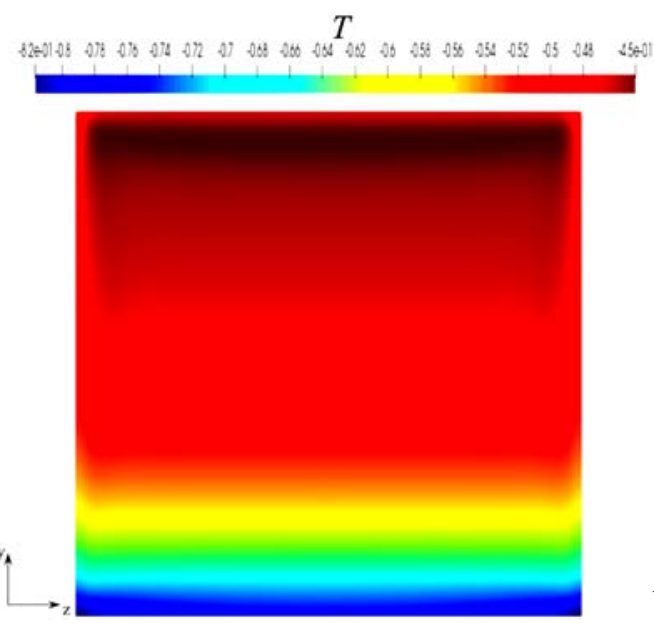

b)

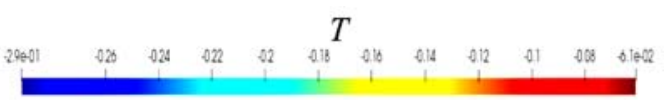

c)

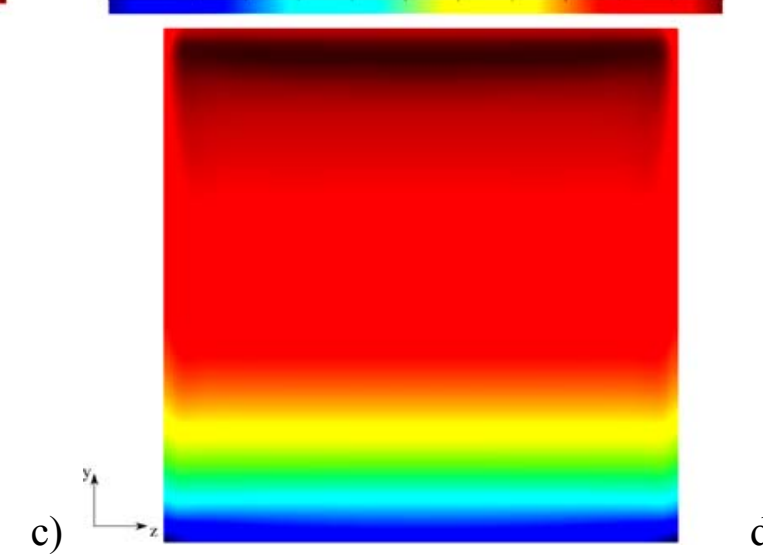

Figure 20: Temperature distribution in the yz mid-plane: a) $\theta=90^{\circ}, T_{\text {cold }}=20^{\circ} \mathrm{C}, \Delta T=20^{\circ} \mathrm{C}$, $B i=0.0526, \mathrm{~b}) \theta=90^{\circ}, T_{\text {cold }}=60^{\circ} \mathrm{C}, \Delta T=20^{\circ} \mathrm{C}, B i=0.526$, c) $\left.\theta=40^{\circ}, T_{\text {cold }}=60^{\circ} \mathrm{C}, \Delta T=20^{\circ} \mathrm{C}, B i=0.526, \mathrm{~d}\right)$ $\theta=40^{\circ}, T_{\text {cold }}=60^{\circ} \mathrm{C}, \Delta T=40^{\circ} \mathrm{C}, B i=0.526$. 
While Fig. 20 finally illustrates the temperature distribution in the yz midplane for different 3D cases, Tables VII-XI provide a summary of all cases considered in the present work from both qualitative (see Tables VI and VII about the spatio-temporal behavior) and quantitative points of view (in terms of the Nusselt number, Tables VIII-XI).

Table VI: Summary of numerical simulations and experiments for the case with temperature difference $\left(\Delta T=20^{\circ} \mathrm{C}\right)$ perpendicular to gravity. Legend: $\mathrm{S}$ (Steady state), OS (Oscillatory State).

\begin{tabular}{lllllll}
\hline \hline$\theta=90^{\circ}$, & $2 \mathrm{D}$ & $2 \mathrm{D}$ & $2 \mathrm{D}$ & Exp & $3 \mathrm{D}$ & $3 \mathrm{D}$ \\
$T_{\text {amb }} \cong 22^{\circ} \mathrm{C}$ & $B i=0.0526$ & $B i=0.2632$ & $B i=0.526$ & & $B i=0.0526$ & $B i=0.526$ \\
\hline \hline$T_{\text {cold }}=20^{\circ} \mathrm{C}$ & $(\mathrm{S})$ & $(\mathrm{S})$ & $(\mathrm{S})$ & $(\mathrm{S})$ & $(\mathrm{S})$ & - \\
$T_{\text {cold }}=40^{\circ} \mathrm{C}$ & $(\mathrm{S})$ & $(\mathrm{S})$ & $(\mathrm{S})$ & $(\mathrm{S})$ & - & - \\
$T_{\text {cold }}=60^{\circ} \mathrm{C}$ & $(\mathrm{S})$ & {$[\mathrm{OS}]$} & {$[\mathrm{OS}]$} & $(\mathrm{S})$ & - & $(\mathrm{S})$ \\
\hline \hline
\end{tabular}

Table VII: Summary of numerical simulations and experiments for the inclined configuration ( $\Delta T=20^{\circ} \mathrm{C}$ with the exception of the starred $3 \mathrm{D}$ case for which $\Delta T=40^{\circ} \mathrm{C}$ ). Legend: $\mathrm{S}$ (Steady state), OS (Oscillatory State), $\mathrm{CH}$ (Chaotic state, the double asterisk refers to a case for which multiple solutions have been found).

\begin{tabular}{lllllll}
\hline \hline$\theta=40^{\circ}$, & $2 \mathrm{D}$ & $2 \mathrm{D}$ & $2 \mathrm{D}$ & Exp & $3 \mathrm{D}$ & $3 \mathrm{D}^{*}\left(\Delta T=40^{\circ} \mathrm{C}\right)$ \\
$T_{\text {amb }} \cong 22^{\circ} \mathrm{C}$ & $B i=0.0526$ & $B i=0.2632$ & $B i=0.526$ & & $B i=0.526$ & $B i=0.526$ \\
\hline \hline$T_{\text {cold }}=20^{\circ} \mathrm{C}$ & $(\mathrm{S})$ & $\{\mathrm{CH}\}$ & {$[\mathrm{OS}]$} & $(\mathrm{S})$ & - & - \\
$T_{\text {cold }}=40^{\circ} \mathrm{C}$ & $\{\mathrm{CH}\}$ & $\{\mathrm{CH}\}$ & $\{\mathrm{CH}\}$ & $(\mathrm{S})$ & - & - \\
$T_{\text {cold }}=60^{\circ} \mathrm{C}$ & $\{\mathrm{CH}\}$ & $\{\mathrm{CH}\}$ & $\{\mathrm{CH}\}^{* *}$, & $(\mathrm{S})$ & $(\mathrm{S})$ & {$[\mathrm{OS}]$} \\
\hline \hline
\end{tabular}

Table VIII: Summary of 2D numerical simulations for the case with temperature difference $\left(\Delta T=20^{\circ} \mathrm{C}\right)$ perpendicular to gravity. The Nusselt number has been evaluated for all vertical (hot and cold) and horizontal (top and bottom) boundaries where the solid is in contact with the liquid; positive and negative values indicate heat entering or leaving the liquid, respectively.

\begin{tabular}{ccccccc}
\hline \hline$T_{\text {cold }}$ & \multicolumn{2}{c}{$B i=0.0526$} & \multicolumn{2}{c}{$B i=0.263$} & \multicolumn{2}{c}{$B i=0.526$} \\
\hline \hline \multirow{2}{*}{$20^{\circ} \mathrm{C}$} & $N u_{\text {Hot }}$ & 1.171 & $N u_{\text {Hot }}$ & 1.278 & $N u_{\text {Hot }}$ & 1.342 \\
& $N u_{\text {Cold }}$ & -1.084 & $N u_{\text {Cold }}$ & -0.9614 & $N u_{\text {Cold }}$ & -0.8837 \\
& $N u_{\text {Top }}$ & -0.07099 & $N u_{\text {Top }}$ & -0.2081 & $N u_{\text {Top }}$ & -0.2976 \\
& $N u_{\text {Bottom }}$ & -0.02256 & $N u_{\text {Bottom }}$ & -0.114 & $N u_{\text {Bottom }}$ & -0.1668 \\
\hline \hline \multirow{3}{*}{$40^{\circ} \mathrm{C}$} & $N u_{\text {Hot }}$ & 1.257 & $N u_{\text {Hot }}$ & 1.638 & $N u_{\text {Hot }}$ & 1.873 \\
& $N u_{\text {Cold }}$ & -0.946 & $N u_{\text {Cold }}$ & -0.553 & $N u_{\text {Cold }}$ & -0.3049 \\
& $N u_{\text {Top }}$ & -0.1792 & $N u_{\text {Top }}$ & -0.6003 & $N u_{\text {Top }}$ & -0.871 \\
& $N u_{\text {Bottom }}$ & -0.1356 & $N u_{\text {Bottom }}$ & -0.491 & $N u_{\text {Bottom }}$ & -0.6978 \\
\hline \hline \multirow{3}{*}{$60^{\circ} \mathrm{C}$} & $N u_{\text {Hot }}$ & 1.342 & $N u_{\text {Hot }}$ & 1.983 & $N u_{\text {Hot }}$ & 2.386 \\
& $N u_{\text {Cold }}$ & -0.8179 & $N u_{\text {Cold }}$ & -0.1643 & $N u_{\text {Cold }}$ & 0.2441 \\
& $N u_{\text {Top }}$ & -0.2836 & $N u_{\text {Top }}$ & -0.982635 & $N u_{\text {Top }}$ & -1.45 \\
& $N u_{\text {Bottom }}$ & -0.241 & $N u_{\text {Bottom }}$ & -0.4908 & $N u_{\text {Bottom }}$ & -1.194 \\
\hline \hline
\end{tabular}


Table IX: Summary of 3D numerical simulations for the case with temperature difference ( $\Delta T=20$ ${ }^{\circ} \mathrm{C}$ ) perpendicular to gravity. The Nusselt number has been evaluated for all (hot and cold, front and back, top and bottom) boundaries where the solid is in contact with the liquid; positive and negative values indicate heat entering or leaving the liquid, respectively.

\begin{tabular}{ccc}
\hline \hline & $N u_{\text {Hot }}$ & 1.499 \\
& $N u_{\text {Cold }}$ & -0.7507 \\
$T_{\text {cold }}=20^{\circ} \mathrm{C}$, & $N u_{\text {Top }}$ & -0.272 \\
$B i=0.0526$ & $N u_{\text {Bottom }}$ & -0.1145 \\
& $N u_{\text {Front }}$ & -0.1621 \\
& $N u_{\text {Back }}$ & -0.1612 \\
\hline \hline & $N u_{\text {Hot }}$ & 3.228 \\
& $N u_{\text {Cold }}$ & 0.9992 \\
$T_{\text {cold }}=60{ }^{\circ} \mathrm{C}$, & $N u_{\text {Top }}$ & -1.299 \\
$B i=0.526$ & $N u_{\text {Bottom }}$ & -0.9628 \\
& $N u_{\text {Front }}$ & -0.9762 \\
& $N u_{\text {Back }}$ & -0.9755 \\
\hline \hline
\end{tabular}

Table X: Summary of 2D numerical simulations for the inclined configuration $\left(\Delta T=20^{\circ} \mathrm{C}\right)$. The Nusselt number has been evaluated for all vertical (hot and cold) and horizontal (top and bottom) boundaries where the solid is in contact with the liquid; positive and negative values indicate heat entering or leaving the liquid, respectively.

\begin{tabular}{ccccccc}
\hline \hline$T_{\text {cold }}$ & \multicolumn{2}{c}{$B i=0.0526$} & \multicolumn{2}{c}{$B i=0.263$} & \multicolumn{2}{c}{$B i=0.526$} \\
\hline \hline \multirow{2}{*}{$20^{\circ} \mathrm{C}$} & $N u_{\text {Hot }}$ & 1.162 & $N u_{\text {Hot }}$ & 1.274 & $N u_{\text {Hot }}$ & 1.342 \\
& $N u_{\text {Cold }}$ & -1.072 & $N u_{\text {Cold }}$ & -0.9455 & $N u_{\text {Cold }}$ & -0.873 \\
& $N u_{\text {Top }}$ & -0.05734 & $N u_{\text {Top }}$ & -0.1782 & $N u_{\text {Top }}$ & -0.2581 \\
& $N u_{\text {Bottom }}$ & -0.03741 & $N u_{\text {Bottom }}$ & -0.1463 & $N u_{\text {Bottom }}$ & -0.2145 \\
\hline \hline \multirow{3}{*}{$40^{\circ} \mathrm{C}$} & $N u_{\text {Hot }}$ & 1.253 & $N u_{\text {Hot }}$ & 1.651 & $N u_{\text {Hot }}$ & 1.909 \\
& $N u_{\text {Cold }}$ & -0.9311 & $N u_{\text {Cold }}$ & -0.535 & $N u_{\text {Cold }}$ & -0.2923 \\
& $N u_{\text {Top }}$ & -0.1633 & $N u_{\text {Top }}$ & -0.5705 & $N u_{\text {Top }}$ & -0.8271 \\
& $N u_{\text {Bottom }}$ & -0.151 & $N u_{\text {Bottom }}$ & -0.5451 & $N u_{\text {Bottom }}$ & -0.7947 \\
\hline \hline & $N u_{\text {Hot }} \mathrm{C}$ & 1.341 & $N u_{\text {Hot }}$ & 2.022 & $N u_{\text {Hot }}$ & 2.49 \\
& $N u_{\text {Cold }}$ & -0.8 & $N u_{\text {Cold }}$ & -0.1586 & $N u_{\text {Cold }}$ & 0.2235 \\
& $N u_{\text {Top }}$ & -0.269 & $N u_{\text {Top }}$ & -0.9447 & $N u_{\text {Top }}$ & -1.369 \\
& $N u_{\text {Bottom }}$ & -0.2585 & $N u_{\text {Bottom }}$ & -0.9231 & $N u_{\text {Bottom }}$ & -1.353 \\
\hline \hline
\end{tabular}


Table XI: Summary of 3D numerical simulations for the inclined configuration with temperature difference. The Nusselt number has been evaluated for all (hot and cold, front and back, top and bottom) boundaries where the solid is in contact with the liquid; positive and negative values indicate heat entering or leaving the liquid, respectively.

\begin{tabular}{ccc}
\hline \hline & $N u_{\text {Hot }}$ & 3.3265 \\
$T_{\text {cold }}=60^{\circ} \mathrm{C}$, & $N u_{\text {Cold }}$ & 0.9449 \\
$\Delta T=20^{\circ} \mathrm{C}$, & $N u_{\text {Top }}$ & -1.232 \\
$B i=0.0526$ & $N u_{\text {Bottom }}$ & -1.13078 \\
& $N u_{\text {Front }}$ & -0.9603 \\
& $N u_{\text {Back }}$ & -0.9591 \\
\hline \hline & $N u_{\text {Hot }}$ & 2.314 \\
$T_{\text {cold }}=60^{\circ} \mathrm{C}$, & $N u_{\text {Cold }}$ & 0.1913 \\
$\Delta T=40^{\circ} \mathrm{C}$, & $N u_{\text {Top }}$ & -0.72 \\
$B i=0.526$ & $N u_{\text {Bottom }}$ & -0.6679 \\
& $N u_{\text {Front }}$ & -0.5636 \\
& $N u_{\text {Back }}$ & -0.5635 \\
\hline \hline
\end{tabular}

Some general trends can be recognized in these conclusive tables. As expected, an increase in $B i$, leads to a corresponding increase in the heat being lost through the solid shell (as witnessed by the corresponding variations of $N u_{T o p}$ and $N u_{\text {Bottom }}$ for the $2 \mathrm{D}$ cases and $\left(N u_{\text {Top }}, N u_{\text {Bottom }}, N u_{\text {Front }}, N u_{\text {Back }}\right)$ for the 3D configuration. A similar effect is obtained when $T_{\text {cold }}$ is made higher.

Among other things, these tables are instrumental in demonstrating quantitatively that for a fixed combination of $T_{\text {cold }}$ and $B i$, the amount of heat lost through the non-thermally controlled walls is larger for the 3D case (as witnessed by the increase in the summation $\Phi$ of the related values of $N u$ ). As an example, for $T_{\text {cold }}=20^{\circ} \mathrm{C}$ and $B i=0.0526$ (horizontal configuration) $\Phi_{2 \mathrm{D}}=N u_{T o p}+N u_{B o t t o m}$ $\cong 0.09$ and $\Phi_{3 \mathrm{D}}=N u_{T o p}+N u_{\text {Bottom }}+N u_{\text {Front }}+N u_{\text {Back }} \cong 0.7$ (3D). Similarly, for $T_{\text {cold }}=60^{\circ} \mathrm{C}$ and $B i=0.526$ (yet for horizontal configuration) $\Phi_{2 \mathrm{D}} \cong 2.6$ and $\Phi_{3 \mathrm{D}} \cong 4.2$ (3D). For $T_{\text {cold }}=60^{\circ} \mathrm{C}$ and $B i=0.526$ (inclined configuration) $\Phi_{2 \mathrm{D}} \cong 2.7$ and $\Phi_{3 \mathrm{D}} \cong 4.3(3 \mathrm{D})$.

Interestingly, these tables also reveal that for the largest value of $T_{\text {cold }}$ and $B i$ considered $\left(60^{\circ} \mathrm{C}\right.$ and 0.526 , respectively) the solid wall where the external temperature is $T_{\text {cold }}$, can become a source of heat for the fluid (owing to the big temperature difference established with respect to the external environment). This is reflected by the positive value taken by $N u_{\text {Cold }}$ in this specific case.

\section{Conclusions}

Thermal convection is a canonical subject of study in the general field of non-linear dynamics, of great interest due to the non-trivial connections existing between the system initial and boundary conditions and the 'emerging states'. This type of convection is also extremely sensitive to the relative orientation of gravity and the direction of the prevailing temperature gradient. While the dependence on initial and boundary conditions can force the system to select different branches of 
solution existing in the phase space 'in parallel', the inclination of the temperature gradient to gravity can exert an indirect influence on these branches (determining their existence and effective location in the space of parameters). These peculiar properties often represent a bottleneck limiting the validity and generality of experimental studies (as even minute changes in the effective conditions established during the experiments can cause remarkable variations in the evolutionary path taken by these systems). The interrelations among all these influential factors is often not easy to discern, therefore, making difficult the elaboration of precise guidelines relating to physical factors that can increase the probability of getting steady or time-dependent flow in practical applications.

In order to fix the great deal of controversy that exists in this field, often due to misinterpretation of the observed dynamics, the first objective of the present work was to identify and address some shortcomings of the existing orthodoxy based on the paradigms of adiabatic or conducting walls. Though these classical boundary conditions have led to a great amount of valuable knowledge, more sophisticated models are needed to predict and estimate effects emerging due to the departure of real configurations from idealized situations.

Along these lines, our research involved sifting through existing studies with different foci in order to glean hints and draw inferences about the factors which may cause a departure from known behaviors.

In order to explore the role of thermal boundary conditions, in the present study, we have deliberately focused on a common fluid (water) encapsulated into a shell displaying homogeneous and isotropic properties along all directions with the external walls subjected to 'active' thermal control (differentially heated walls, maintained at fixed temperature) or naturally undergoing heat exchange with the external environment.

For the purpose of specifically identifying and quantifying 3D effects, we followed a logical process with systems of increasing complexity being addressed progressively, i.e. 2D numerical simulations, followed by experiments and 3D simulations. Three-dimensional simulations have expressly been used to gain further understanding of the considered dynamics via cross comparison of such computations with reality (experiments) and idealized behaviors (2D flow).

Such approach has provided important clues regarding the nature of the observed effects. In particular, the simulations initially conducted under the constraint of two-dimensionality have been instrumental in showing that, as a result of a departure from idealized boundary conditions, significant modifications can occur in the typical route followed by these systems in their evolution towards chaos. By increasing the rate of heat exchange with the external environment (via an increase in $T_{\text {cold }}$ or $B i$ for a fixed $T_{\text {amb }}$ ) transition to oscillatory states or chaos can be promoted, which is a relatively counter-intuitive finding.

This trend can be justified according to the modes of RB nature that are excited when the horizontal boundaries are not adiabatic, as also witnessed by the proliferation of these modes when the cavity is inclined and, accordingly, a vertical (destabilizing) component of the temperature gradient is naturally produced in the fluid. Vice versa, when the inclined configuration is gradually turned into 
the classical Hadley flow (by making the imposed temperature difference perfectly perpendicular to gravity), chaotic states are progressively replaced by more regular solutions (steady or simply timeperiodic) and multiple solutions tend to be suppressed at all.

If $3 \mathrm{D}$ configurations are considered (via experiments or 3D computations), strong stabilization of the flow occurs. These effects generally lead to an increase in the value of the temperature difference required for flow instability and can even cause complete re-stabilization of the flow in many circumstances (with the emergence of steady states in wide regions of the space of parameters). We could directly ascribe this trend to the heat loss taking place to the vertical boundaries (delimiting the system along the third direction, i.e. in the spanwise direction) where temperature is not actively controlled.

In the light of these results, we conclude that solid walls exchanging heat with the external environment can have a diametrically opposite role in influencing the flow stability depending on their relative orientation with respect to gravity. While vertical walls where temperature is not actively controlled simply contribute to energy extraction, thereby promoting system stabilization, horizontal walls can bring in new fluid-dynamic disturbances.

We sincerely hope that the present study will help produce new strategies that will greatly support downstream implementation of new models addressing the causes of uncertainty in the (numerical) prediction of the effective states of thermal convection and related transition to turbulence.

Future efforts shall also be devoted to consider the situation in which the imposed temperature gradient and gravity are perfectly parallel (corresponding to the classical Rayleigh-Bénard paradigm). As the number of potential instabilities and the multiplicity of flow fields is known to increase dramatically in this case (even though the canonical paradigms of adiabatic or isothermal walls are considered), it is expected that more realistic boundary conditions may lead to a variety of new solutions, thereby originating a new line of inquiry for this very classical subject.

\section{References}

[01] Melnikov D.E. and Shevtsova V.M., (2005), "Liquid Particles Tracing in Three-dimensional Buoyancy-driven Flows", Fluid Dyn. Mater. Process., 1(2): 189-200.

[02] Kaddeche, S., Garandet, J.P., Henry, D., Hadid, H.B., Mojtabi, A.,2015,On the effect of natural convection on solute segregation in the horizontal Bridgman configuration: Convergence of a theoretical model with numerical and experimental data, J. Cryst. Growth 409, 89-94.

[03] Delgado-Buscalioni R., Crespo del Arco E., Bontoux P., Ouazzani J., (1998), "Convection and instabilities in differentially heated inclined shallow rectangular boxes", Compte Rend. Acad. Sci.. II B (Mechanics-Physics-Astronomy), 326(11): 711-718.

[04] Sun Z. and Jaluria Y., (2011), Conjugate thermal transport in gas flow in long rectangular microchannel, J. Electron. Packag. 133(2), 021008 (11 pages).

[05] Martineau R.C., Berry R.A., Esteve A., Hamman K.D., Knoll D.A., Park R., Taitano W., (2010), Comparison of natural convection flows under VHTR type conditions modeled by both the conservation and incompressible forms of the Navier-Stokes equations, Nucl. Eng. Des. 240, 13711385. 
[06] Bontoux P., Roux B., Schiroky G. H., Markham B. L., and Rosenberger F., (1986), Convection in the vertical midplane of a horizontal cylinder. Comparison of two-dimensional approximations with three-dimensional results, Int. J. Heat Mass Transfer, 29(2), 227-240.

[07] Bennacer, R., El Ganaoui, M., Maré, T., Nguyen, C.T. (2009), Natural convection of nanofluids in a cavity including the soret effect, Computational Thermal Sciences, 1(4), pp. 425440

[08] Hadidi N., and Bennacer R., (2016), Three-dimensional double diffusive natural convection across a cubical enclosure partially filled by vertical porous layer, Int. J. Therm. Sci., 101, 143-157

[09] Sohail, M. and Saghir, M.Z., (2006), Three-Dimensional Modeling of the Effects of Misalignment on the Growth of Ge1-xSix by The Traveling Solvent Method, Fluid Dyn. Mater. Process., 2 (2), 127-140.

[10] Lappa M., (2012), Rotating Thermal Flows in Natural and Industrial Processes, John Wiley \& Sons, Ltd (2012, Chichester, England).

[11] Soltani N.and Rahal S., (2017), Control of the Convective Flow Instabilities in a Simulated Czochralski Growth System, FDMP: Fluid Dynamics \& Materials Processing, 13(1) 1-17, doi:10.3970/fdmp.2017.013.001

[12] Azoui H., Bahloul D. and Soltani N., (2018), Three-Dimensional Numerical Investigation of Convective Thermal Instabilities in the Sapphire Melt for Czochralski Growth Process, FDMP:

Fluid Dynamics \& Materials Processing, 14(2),.87-105, doi: 10.3970/fdmp.2018.01149

[13] Hadley G., (1735), "Concerning the cause of the general trade winds", Phil. Trans. Roy. Soc. Lond., 29: 58-62.

[14] Cross M.C. and Hohenberg P.C., (1993), "Pattern formation outside of equilibrium", Rev. Mod. Phys., 65: 851-1112.

[15] Emanuel K. A., (1991), "The Theory of Hurricanes", Annual Review of Fluid Mechanics, 23: $179-196$.

[16] Thorpe, S. A., (1987) Transitional phenomena and the development of turbulence in stratified fluids: A review, J. Geophys. Res. 92C, 5231-5248.

[17] Richter, F.M., (1973) Convection and the large-scale circulation of the mantle, J. Geophys. Res., 78, 8735-8745

[18] Woods A.W., and Lintz, S.J. (1992) Natural convection and dispersion in a tilted fracture, $J$. Fluid Mech., 241, 59-74.

[19] Heng K. and Showman A.P., (2015), "Atmospheric Dynamics of Hot Exoplanets", Annual Review of Earth and Planetary Sciences, 43: 509-540.

[20] Lappa M., (2005), "On the nature and structure of possible three-dimensional steady flows in closed and open parallelepipedic and cubical containers under different heating conditions and driving forces", Fluid Dynamics \& Materials Processing, 1(1): 1-19. DOI: 10.3970/fdmp.2005.001.001

[21] Lappa M., (2007), "Secondary and oscillatory gravitational instabilities in canonical threedimensional models of crystal growth from the melt, Part2: Lateral heating and the Hadley circulation", Comptes Rendus Mécanique, 335(5-6): 261-268. DOI:10.1016/j.crme.2007.05.004

[22] Lappa M., (2017), On the oscillatory hydrodynamic modes in liquid metal layers with an obstruction located on the bottom, Int. J. Thermal Science, 118: 303-319. DOI: 10.1016/j.ijthermalsci.2017.04.013

[23] Gelfgat A.Yu., Bar-Yoseph P.Z. and Yarin A.L., (1999b), "Stability of Multiple Steady States of Convection in Laterally Heated Cavities", J. Fluid Mech.., 388: 315-334.

[24] Benoit M.R., Brown R.B., Todd P., Nelson E.S. and Klaus D.M., (2008), "Buoyant plumes from solute gradients generated by non-motile Escherichia coli”, Physical Biology, 5(4), 046007 (10pp).

[25] Lappa M., (2009), Thermal Convection: Patterns, Evolution and Stability, John Wiley \& Sons, Ltd (2009, Chichester, England). 
[26] Hart J.E., (1972), Stability of thin non-rotating Hadley circulations,. J. Atmos. Sci., 29: 687697.

[27] Hart J.E., (1983), "A note on the stability of low-Prandtl-number Hadley circulations", J. Fluid Mech., 132: 271-281.

[28] Gill A. E., (1974), "A theory of thermal oscillations in liquid metals", J. Fluid Mech. 64 (3): 577-588.

[29] Kuo H.P., Korpela S.A., (1988), "Stability and finite amplitude natural convection in a shallow cavity with insulated top and bottom and heated from the side", Phys. Fluids, 31: 33-42.

[30] Gershuni G.Z., Laure P., Myznikov V.M., Roux B., Zhukhovitsky E.M., (1992), "On the stability of plane-parallel advective flows in long horizontal layers", Microgravity Q., 2(3): 141-151. [31] Delgado-Buscalioni R., (2001), "Convection patterns in end-heated inclined enclosures" Phys. Rev. E 64, 01630317 pages

[32] Hart J.E., (1970), "Stability of the flow in a differentially heated inclined box", J. Fluid Mech. 47(3): 547-576.

[33] Rohsenow W., Hartnet J., Cho Y., (1998). Handbook of Heat Transfer (3rd ed.). McGraw-Hill. [34] Lappa M., (1997), "Strategies for parallelizing the three-dimensional Navier-Stokes equations on the Cray T3E"; Science and Supercomputing at CINECA, 11, 326-340. ISBN-10: 88-86037-031, M. Voli, Editor (Bologna, Italy).

[35] Lappa M., (2019), A mathematical and numerical framework for the simulation of oscillatory buoyancy and Marangoni convection in rectangular cavities with variable cross section, Chapter 12 (pp. 419-458) in Computational Modeling of Bifurcations and Instabilities in Fluid Mechanics (Springer Mathematical Series, 2018, Part of the Computational Methods in Applied Sciences book series - COMPUTMETHODS, volume 50), Editor Alexander Gelfgat - ISBN 978-3-319-91493-0. DOI: 10.1007/978-3-319-91494-7 https://www.springer.com/fr/book/9783319914930

[36] Ladyzhenskaya O.A., (1969), The Mathematical Theory of Viscous Incompressible Flow, Gordon and Breach, 2nd Edition, New York - London, 1969.

[37] Patankar S.V.,(1981),"Numerical heat transfer and fluid flow”, Hemisphere Publish-ing Corporation, 1981.

[38] Issa R.I.,(1986), "Solution of the implicitly discretized fluid flow equations by operatorsplitting", J. Comp. Physics, 62:40-65.

[39] Rhie C.M. and Chow W.L., (1983), Numerical study of the turbulent flow past an airfoil with trailing edge separation, AIAA J 21,1525-1532

[40] De Vahl Davis and Jones I. P., "Natural convection in a square cavity a comparison exercise", International journal for numerical methods in fluids, 3, 227-248.

[41] Bennett B. A. V. and Hsueh J., (2006), Natural Convection in a Cubic Cavity: Implicit Numerical Solution of Two Benchmark Problems, Numerical Heat Transfer, Part A: Applications, 50:2, 99-123.

[42] Fusegi T., Hyun J. M., Kuwahara K., and Farouk B., (1991), A Numerical Study of ThreeDimensional Natural Convection in a Differentially Heated Cubical Enclosure, Int. J. Heat Mass Transfer, 34, 1543-1557.

[43] Tric E., Labrosse G., and Betrouni M., (2000), A First Incursion into the 3D Structure of Natural Convection of Air in a Differentially Heated Cubic Cavity, from Accurate Numerical Solutions, Int. J. Heat Mass Transfer, 43, 4043-4056.

[44] Wakashima S. and Saitoh T. S., (2004), Benchmark Solutions for Natural Convection in a Cubic Cavity Using the High-Order Time-Space Method, Int. J. Heat Mass Transfer, 47, 853-864.

[45] Torres J.F., Henry D., Komiya A., Maruyama S, (2015), "Transition from multiplicity to singularity of steady natural convection in a tilted cubical enclosure", Phys. Rev. E, 92, 023031.

[46] Wang Q., Wan Z., Yan R., Sun D., (2018), "Multiple states and heat transfer in twodimensional tilted convection with large aspect ratios", Phys. Rev. Fluids, 3, 113503. 
[47] Le Quéré P., and Penot F., (1987), "Numerical and Experimental investigation of the transition to unsteady natural convection of air in a vertically differentially heated cavity", ASME HTD, 94: $75-82$.

[48] Henkes R.A.W.M. and Hoogendoorn C.J., (1990), "On the stability of the natural convection flow in a square cavity heated from the side", Applied Scientific Research, 47(3): 195 - 220 .

[49] Bucchignani E., (2009), "An Implicit Unsteady Finite Volume Formulation for Natural Convection in a Square Cavity", Fluid Dyn. Mater. Process., 5(1): 37-60.

[50] Yahata H., (1999), "Stability Analysis of Natural Convection in Vertical Cavities with Lateral Heating ", J. Phys. Soc. Jpn., 68(2): 446-460

[51] Paolucci S. and Chenoweth D.R., (1989), "Transition to chaos in a differentially heated vertical cavity", J. Fluid Mech., 201: 379-410.

[52] Paolucci S., (1990), "Direct simulation of two-dimensional turbulent natural convection in an enclosed cavity", J. Fluid Mech., 215: 229-262.

[53] Janssen R.J.A., and Henkes R.A.W.M., (1995), "Influence of Prandtl number on instability mechanisms and transition in a differentially heated square cavity", J. Fluid Mech., 290 : 319-344.

[54] Xin S. and Le Quéré P., (1995), "Direct numerical simulations of two-dimensional chaotic natural convection in a differentially heated cavity of aspect ratio 4", J. Fluid Mech., 304, 87-118.

[55] Farhangnia M., Biringen S, Peltier L. J., (1996), "Numerical Simulation of Two-dimensional Buoyancy-driven Turbulence in a Tall Rectangular Cavity", Int. J. Numer. Meth. Fluids, 23(12): $1311-1326$.

[56] Yahata H., (1997), "Thermal Convection in a Vertical Slot with Lateral Heating", J. Phys. Soc. Jpn., 66(11): 3434-3443.

[57] Le Quéré P. and Behnia M., (1998), "From onset of unsteadiness to chaos in a differentially heated square cavity", J. Fluid Mech., 359: 81-107.

[58] Mayne D.A., Usmani A.S., Crapper M., (2000), "h-Adaptive finite element solutions of high Rayleigh number thermally driven cavity problem", Int. J. Num. Meth. Heat Fluid Flow, 10: 598615.

[59] TianY.S. and Karayiannis T. G., (2000), "Low Turbulence Natural Convection in an Air Filled Square Cavity. Part I: The thermal and fluid flow fields", Int. J. Heat Mass Transfer, 1(43): 849-866. [60] Lappa M., (2016), A Mathematical and Numerical Framework for the Analysis of Compressible Thermal Convection in Gases at very high Temperatures, Journal of Computational Physics, 313: 687-712, DOI:10.1016/j.jcp.2016.02.062

[61] Ravi M.R., Henkens R.A.W.M. and Hoogendoorn C.J., (1994), "On the high Rayleigh-number structure of steady natural convection flow in a square enclosure", J. Fluid Mech., 262: 325-351

[62] Jones D.N. and Briggs D.G., (1989), "Periodic Two-Dimensional Cavity Flow: Effect of Linear Horizontal Thermal Boundary Condition", J. Heat Transfer, 111: 86-91.

[63] Winters K.H., (1987), "Hopf bifurcation in the double glazing problem with conducting boundaries", J. Heat Transfer, 109: 894-898.

[64] Gelfgat A.Yu. and Tanasawa I., (1994), "Numerical analysis of oscillatory instability of buoyancy convection with the Galerkin spectral method", Numer. Heat Transfer, Part A, 25(6): 627-648.

[65] Xin S. and Le Quéré P., (2001), "Linear stability analyses of natural convection in a differentially heated square cavity with conducting horizontal walls", Phys. Fluids, 13(9): 25292542.

[66] Lappa M., and Gradinscak T., (2018), On the Oscillatory Modes of Compressible Thermal Convection in inclined differentially heated cavities, Int. J. of Heat and Mass Transfer, 121: 412436. DOI: 10.1016/j.ijheatmasstransfer.2017.12.095

[67] Wiliamson N., Armfield S.W., Lin W., Kirkpatrick M.P., (2016), "Stability and Nusselt number scaling for inclined differentially heated cavity flow”, Int. J. Heat Mass Transfer, 97: 787793. 
[68] Yang, H.Q., Yang, K.T. and Lloyd, J.R. (1987) Laminar natural-convection flow transitions in tilted three-dimensional longitudinal rectangular enclosures, Int. J. Heat Mass Transfer, 30 (8), 1637-1644.

[69] Yang, H.Q., Yang, K.T. and Lloyd, J.R. (1988) Three-dimensional bimodal buoyant flow transitions in tilted enclosures, Int. J. Heat Fluid Flow, 9 (2), 90-97.

[70] Lock, G.S.H. and Han, J.-C. (1990) The effects of tilt, skew and roll on natural convection in a slender, laterally-heated cavity, Math. Comput. Model., 13 (2), 23-32.

[71] Catton I., Ayyaswamy P.S., R.M. Clever, (1973), "Natural convection flow in a finite, rectangular slot arbitrarily oriented with respect to the gravity vector", Int. J. Heat Mass Transfer, 17:173-184.

[72] Kuyper R.A., Van Der Meer T.H., Hoogendoorn C.J., Henkes R.A.W.M, (1993), "Numerical study of laminar and turbulent natural convection in an inclined square cavity", Int. J. Heat Mass Transfer, 36(11): 2899-2911.

[73] Adachi T. and Mizushima J., (1996), "Stability of the Thermal Convection in a Tilted Square Cavity", J. Phys. Soc. Jpn.. 65(6): 1686-1698

[74] Lappa M., and Ferialdi H., (2017), On the Oscillatory Hydrodynamic Instability of Gravitational Thermal Flows of Liquid Metals in Variable Cross-section Containers, Physics of Fluids, 29(6), 064106 (19 pages).

[75] Lappa M., and Ferialdi H., (2018a), Gravitational Thermal Flows of Liquid Metals in 3D Variable Cross-section Containers: Transition from low-dimensional to high-dimensional chaos, Chaos, 28, 093114 (21 pages).

[76] Lappa M., and Ferialdi H., (2018b), Multiple solutions, Oscillons and Strange Attractors in ThermoViscoElastic Marangoni Convection, Physics of Fluids, 30(10), 104104 (19 pages).

[77] Lappa M., and Ferialdi H. (2019), Oscillatory and Turbulent Flows of Liquid Metals in Differentially Heated Systems with Horizontal and Non-Horizontal Walls, Chapter 3 (pp. 151-209) in Recent Studies in Materials Science, Patrick R. Lind Editor, Nova Science Publishers Inc., Series: Materials Science and Technologies, ISBN: 978-1-53615-270-8. https://novapublishers.com/shop/recent-studies-in-materials-science/

[78] Wang Q., Xia S., Wang B., Sun D., Zhou Q., Wan Z., (2018), "Flow reversals in twodimensional thermal convection in tilted cells", J. Fluid Mech. 849: 355-372.

[79] Schladow S. G., Patterson J. C., and Street R. L., (1989), "Transient Flow in a Side-Heated Cavity at High Rayleigh Number: A Numerical Study, J. Fluid Mech., 200: 121-148.

[80] Kowalewski T.A., (1998), Experimental validation of numerical codes in thermally driven flows, in Adv. in Computational Heat Transfer, pp.1-15, Begel House Inc., NY 1998.

[81] Lee T.L. and Lin T.F., (1995), "Three-Dimensional Natural Convection of Air in an Inclined Cubic Cavity”, Numer. Heat Transfer A, 27: 681-703.

[82] Belmonte A., Tilgner A. and Libchaber A., (1995), "Turbulence and internal waves in sideheated convection", Phys. Rev. E, 51(6): 5681-5687.

[83] Fusegi T., Hyun J.M. and Kuwahara K., (1991), "Three-dimensional simulations of natural convection in a sidewall-heated cube", Int. J. Numer. Meth. Fluids, 13: 857-867. 